\title{
New insights of the correlation between AXIN2 polymorphism and cancer risk and susceptibility: evidence from 72 studies
}

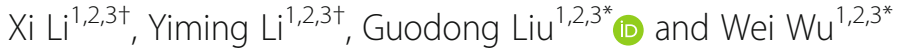

\begin{abstract}
Background: Numerous studies have reported the correlation between AXIN2 polymorphism and cancer risk, but the results seem not consistent. In order to get an overall, accurate and updated results about AXIN2 polymorphism and cancer risk, we conducted this study.

Methods: An updated analysis was performed to analyze the correlation between AXIN2 polymorphisms and cancer risk. Linkage disequilibrium (LD) analysis was also used to show the associations.

Results: Seventy-two case-control studies were involved in the study, including 22,087 cases and 18,846 controls. The overall results showed $r 511079571$ had significant association with cancer risk (allele contrast model: OR $=0.539$, $95 \% \mathrm{Cl}=0.478-0.609$, PAdjust $=0.025$; homozygote model: $\mathrm{OR}=0.22,95 \% \mathrm{Cl}=0.164-0.295$, PAdjust $<0.001$; heterozygote model: $\mathrm{OR}=0.292,95 \% \mathrm{Cl}=0.216-0.394$, PAdjust< 0.001 ; dominant model: $\mathrm{OR}=0.249,95 \% \mathrm{Cl}=$ $0.189-0.33$, PAdjust< 0.001 ). The same results were obtained with rs 1133683 in homozygote and recessive models (PAdjust< 0.05), and in rs35285779 in heterozygote and dominant models (PAdjust< 0.05 ). LD analysis revealed significant correlation between rs7210356 and rs9915936 in the populations of CEU, CHB\&CHS, ESN and JPT (CEU: $r^{2}=0.91$; CHB\&CHS: $r^{2}=0.74$; ESN: $r^{2}=0.62$, JPT: $r^{2}=0.57$ ), and a significant correlation between $r 59915936$ and rs7224837 in the populations of CHB\&CHS, ESN and JPT $\left(r^{2}>0.5\right)$, between rs7224837 and rs7210356 in the populations of CEU, CHB\&CHS, JPT $\left(r^{2}>0.5\right)$, between $r 35435678$ and $r 535285779$ in the populations of CEU, CHB\&CHS and JPT $\left(r^{2}>0.5\right)$.
\end{abstract}

Conclusions: AXIN2 rs11079571, rs1133683 and rs35285779 polymorphisms have significant correlations with overall cancer risk. What's more, two or more polymorphisms such as rs7210356 and rs9915936, rs9915936 and rs7224837, rs7224837 and rs7210356, rs35435678 and rs35285779 have significant correlation with cancer susceptibility in different populations.

Keywords: AXIN2, Polymorphism, Cancer, Analysis, Correlation

\footnotetext{
*Correspondence: guodongliu@csu.edu.cn; wwtw1972@126.com

${ }^{+} \mathrm{Xi}$ Li and Yiming Li contributed equally to this work.

'Department of Geriatric Surgery, Xiangya Hospital, Central South University, Changsha 410008, China

Full list of author information is available at the end of the article
}

(C) The Author(s). 2021 Open Access This article is licensed under a Creative Commons Attribution 4.0 International License, which permits use, sharing, adaptation, distribution and reproduction in any medium or format, as long as you give appropriate credit to the original author(s) and the source, provide a link to the Creative Commons licence, and indicate if changes were made. The images or other third party material in this article are included in the article's Creative Commons licence, unless indicated otherwise in a credit line to the material. If material is not included in the article's Creative Commons licence and your intended use is not permitted by statutory regulation or exceeds the permitted use, you will need to obtain permission directly from the copyright holder. To view a copy of this licence, visit http://creativecommons.org/licenses/by/4.0/ The Creative Commons Public Domain Dedication waiver (http://creativecommons.org/publicdomain/zero/1.0/) applies to the data made available in this article, unless otherwise stated in a credit line to the data. 


\section{Background}

Cancer is currently one of the most important health problems across the world, and it has been well known as the second most common cause of death in the US. According to reports, the estimated data of Cancer Statistics show that 1,762,450 new cases of cancers will be diagnosed in the US in 2019, and 606,880 deaths will be confirmed [1]. Among which, prostate cancer, lung cancer, bronchus cancer and colorectal cancer will account for the top 4 common types in male cases, and breast, lung and colorectal cancers will be the top 3 most common types in female cases [1]. The data from National Central Cancer Registry of China reported that in 2015, 4292,000 new cancer cases and 2814,000 cancer deaths occurred in China, with lung cancer being the most common incident cancer and the leading cause of cancer death. Stomach, esophageal, and liver cancers were also commonly diagnosed and were identified as leading causes of cancer death [2]. In Europe, there were an estimated 3.91 million new cases of cancer and 1.93 million deaths from cancer in 2018, among which, the female breast, colorectal, lung and prostate cancer were the most common cancer sites [3]. In recent years, many studies have pointed out that genomic types may be closely related to the carcinogenic effects of cancers, one of which is the Axin-related protein, AXIN2 [4-7].

The AXIN2 gene locates at chromosome 17q23-24, which belongs to a heterozygosity region that frequently loss in neuroblastoma, breast cancer, and other cancers $[8,9]$. For the biological function, AXIN2 is a critical regulator in Wnt/ $\beta$-catenin signaling, especially for the stability of $\beta$-catenin, which plays an important role in cell growth, genesis of a number of malignancies, tumor progression and so on. For example, Chen et al. [10] reported that miR-183 could regulate bladder cancer cells growth and apoptosis via targeting AXIN2. A recent report by Chen et al. pointed out that down regulating AXIN2 expression could promote human osteosarcoma cell proliferation [11]. Another paper showed that targeting AXIN2 axis could suppress tumor growth and metastasis in colorectal cancer [12]. As the expression or protein structure may be influenced by gene polymorphism, some studies have taken insights in the correlation between AXIN2 and cancer susceptibility. Otero L et al. reported that rs2240308 polymorphism was associated with colorectal cancer (CRC) and the CRC patients who carried this variation in the AXIN2 gene always had a worse prognosis [13]. Zhong et al. showed that the Axin2-148 C/T polymorphism was significantly associated with a decreased risk of cancer, particularly lung cancer, in Asians and population-based controls [14]. Liu et al. showed that rs11655966, rs3923086 and rs7591 of AXIN2 showed significant associations with papillary thyroid carcinoma (PTC) [15]. However the available results remain inconsistent. For example, E•Pinarbasi et al. [16] reported that $r s 2240308$ polymorphism had no significant correlation with the susceptibility of prostate cancer in the Turkish population, whereas Xu et al. [17] revealed that AXIN2 rs2240308 variants may be associated with decreased cancer susceptibility. At the same time, Dai et al. [18] concluded that AXIN2 rs2240308 polymorphism might decrease the susceptibility of lung and prostate cancers. Thus, we designed this metaanalysis to obtain updated and accurate insight to assess the association between AXIN2 polymorphism and cancer susceptibility.

\section{Methods}

\section{Literature retrieval strategy and eligibility criteria}

Wanfang, CNKI, CBM, EMBASE, Web of Science and PubMed databases were used to search the published papers before July, 2020 by using the keywords and $\mathrm{MeSH}$ terms of 'Axin OR AXIN-2' AND 'carcinoma OR cancer OR tumor' AND 'SNP OR mutation OR polymorphism OR variant'. All publications in English and Chinese were involved, references were also evaluated manually to get more comprehensive studies.

The studies that met the following criteria would be included: (1) case-control studies that were related to the correlation of $A X I N-2$ polymorphism and cancer susceptibility; (2) English or Chinese publications, and (3) genotype frequency were provided directly or indirectly. Conversely, the studies that met the following criteria would be excluded: (1) meta-analysis, reviews, case reports or duplicate publications; (2) data of genotype frequency was not informed; (3) data from cell lines or animals.

\section{Data extraction}

All data were examined by two independent researchers (Li X and Li YM). From which, the first author's name, published data, total number of participants, subtypes like cancer type, source of control and ethnicity, genotyping method, and genotype frequency of the AXIN2 gene polymorphisms in all cases and controls were labeled and calculated. Any disagreement would be reexamined and discussed by the other researchers (Liu G and $\mathrm{Wu} \mathrm{W}$ ) and, if necessary, the author of the publications would be requested to provide more data.

\section{Statistical analysis}

In our study, we used five genetic models to evaluate the correlation of AXIN2 gene polymorphisms and cancer risk, including allele contrast model (B vs. A), homozygote comparison model (BB vs. AA), heterozygote comparison model (BA vs. AA), dominant comparison model (BB + BA vs. $\mathrm{AA})$, and recessive comparison model $(\mathrm{BB}$ vs. $\mathrm{BA}+\mathrm{AA})$. The strength of the association 
was checked by OR with $95 \% \mathrm{CI}$, and the significant statistics was confirmed by Z-test and adjusted by Bonferroni corrections, $P_{\text {Adjust }}=P_{z} * 5$ genetic models [19]. Subtypes like ethnicity, type of cancer and source of control were also evaluated by stratified analysis. The $\mathrm{x}^{2}$-test was assessed to analyze the heterogeneity between studies.

$P<0.1$ meant a significant heterogeneity, and if so, we used the random effects model (DerSimonian and Laird methods) to summarize the data [20]; if not, the fixed effect model (Mantel-Haenszel method) was selected [21]. Hardy-Weinberg equilibrium (HWE) was performed for sensitivity analysis [22]. Begg's funnel plots and Egger's line regression test $[23,24]$ were performed to assess the potential publication bias. STATA software system v12.0 was used to perform statistical analysis. $P \leq 0.05$ was considered as a statistically significant difference.

\section{Linkage disequilibrium (LD) analysis}

The data was acquired from 1000 Genomes Project which contains AXIN2 polymorphisms in the present research. Six groups including CEU (Utah residents with
Northern and Western European ancestry from the CEPH collection), CHS (southern Han Chinese, China), CHB (Han Chinese in Beijing, China), ESN (Esan in Nigeria), YRI (Yoruba in Ibadan, Nigeria) and JPT (Japanese in Tokyo, Japan) were involved in the program. Haploview software was performed to analyze the data, and LD analysis was performed by $\mathrm{r}^{2}$ statistics.

\section{Results}

\section{Details of included studies}

Totally, 24 articles were included in this analysis, which contained 72 case-control studies (Fig. 1). Among which, three studies related to the linkage between rs11079571 polymorphism and cancer susceptibility [25-27], six studies focused on rs1133683 [16, 28-32], six studies concerned about $r s 2240307$ [16, 28-30, 33, 34], 20 studies focused on $r s 2240308$ [15, 16, 28-30, 32-44], four studies focused on $r s 35285779$ [16, 28-30], four studies focused on $r s 35415678$ [16, 28-30], five studies focused on $r s 3923086$ [15, 25, 26, 34, 45], five studies focused on rs3923087 [25, 26, 34, 41, 45], three studies focused on rs4072245 [16, 28, 30], five studies focused on $r s 4791171$

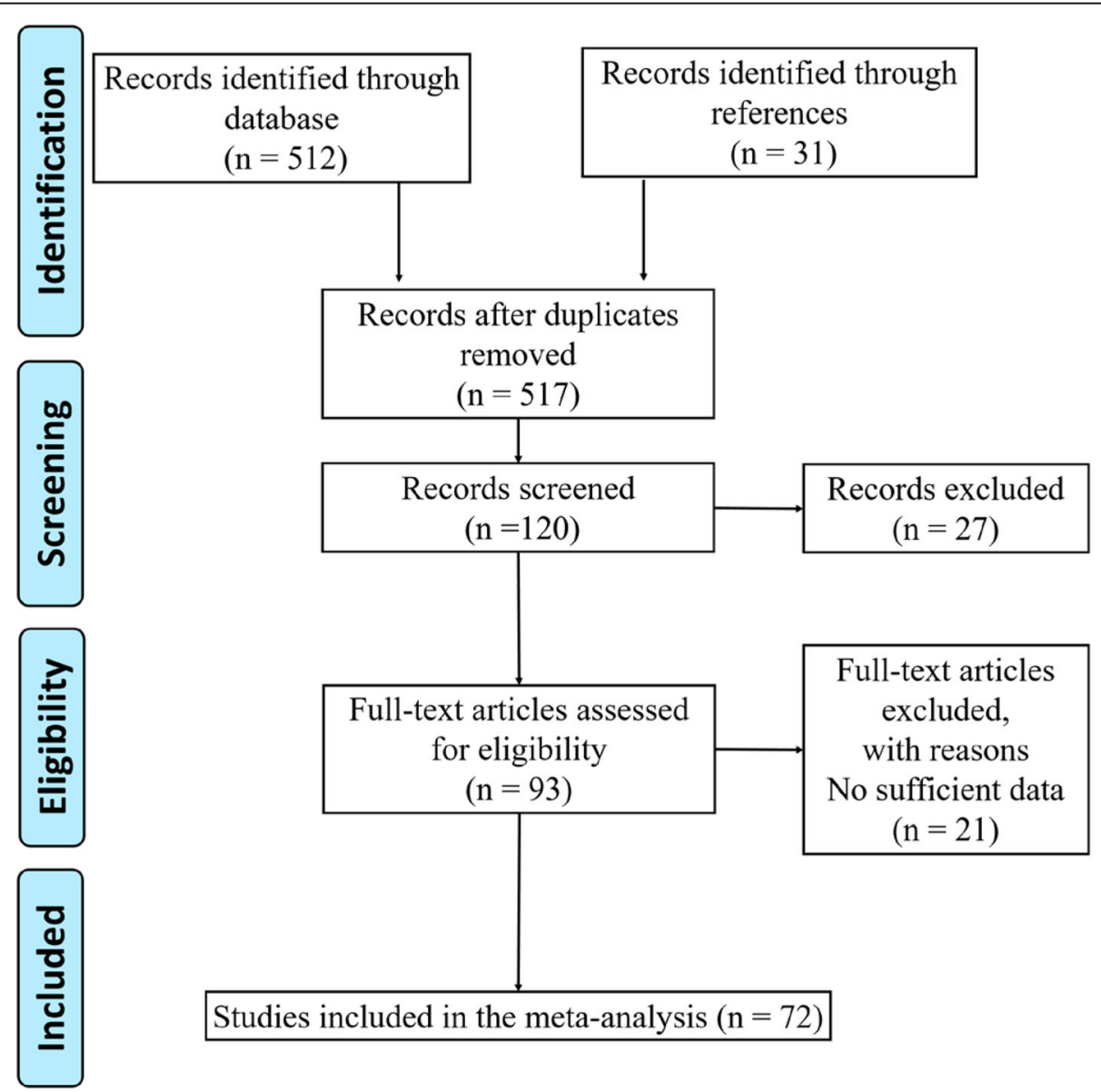

Fig. 1 Flow chart of select methods of the study 
Table 1 Characteristics of the enrolled studies on AXIN2 Polymorphism and cancer

\begin{tabular}{|c|c|c|c|c|c|c|c|c|c|c|c|c|c|}
\hline \multirow[t]{2}{*}{ Polymorphism } & \multirow[t]{2}{*}{ First author } & \multirow[t]{2}{*}{ Year } & \multirow[t]{2}{*}{ Ethnicity } & \multirow{2}{*}{$\begin{array}{l}\text { Genotyping } \\
\text { Method }\end{array}$} & \multirow{2}{*}{$\begin{array}{l}\text { Source } \\
\text { of } \\
\text { Control }\end{array}$} & \multirow[t]{2}{*}{ Cancer Type } & \multicolumn{3}{|c|}{ Cases } & \multicolumn{4}{|c|}{ Controls } \\
\hline & & & & & & & PAA & PAB & PBB & HAA & HAB & $\mathrm{HBB}$ & HWE \\
\hline rs11079571 & Wang et al. & 2008 & Caucasion & GoldenGate & PB & Breast Cancer & 32 & 233 & 533 & 16 & 221 & 606 & Y \\
\hline rs11079571 & Alanazi et al. & 2013 & Asian & TaqMan & PB & Breast Cancer & 182 & 194 & 55 & 11 & 37 & 45 & Y \\
\hline rs11079571 & Zhang et al. & 2015 & Asian & $P C R$ & PB & Acute Leukemia & 196 & 180 & 201 & 42 & 170 & 189 & Y \\
\hline rs1133683 & Gunes et al. & 2009 & Asian & PCR & PB & Lung Cancer & 172 & 204 & 10 & 42 & 50 & 8 & Y \\
\hline rs1133683 & Pinarbasi et al. & 2010 & Asian & PCR & $\mathrm{HB}$ & Prostate Cancer & 724 & 872 & 6 & 44 & 48 & 8 & Y \\
\hline rs1133683 & Gunes et al. & 2010 & Asian & PCR & $\mathrm{HB}$ & Astrocytoma & 70 & 306 & 20 & 42 & 50 & 8 & Y \\
\hline rs1133683 & Davoodi et al. & 2015 & Asian & PCR-RFLP & PB & Ovarian Cancer & 386 & 1210 & 6 & 58 & 34 & 8 & Y \\
\hline rs1133683 & $\begin{array}{l}\text { Rosales-Reynoso } \\
\text { et al. }\end{array}$ & 2016 & Caucasion & PCR-RFLP & PB & $\begin{array}{l}\text { Colorectal } \\
\text { Cancer }\end{array}$ & 124 & 252 & 19 & 22 & 57 & 21 & Y \\
\hline rs1133683 & Bahl et al. & 2017 & Asian & PCR-RFLP & PB & Lung Cancer & 190 & 1406 & 37 & 103 & 169 & 33 & $N$ \\
\hline rs2240307 & Gunes et al. & 2009 & Asian & PCR & PB & Lung Cancer & 96 & 4 & 0 & 95 & 5 & 0 & Y \\
\hline rs2240307 & Pinarbasi et al. & 2010 & Asian & PCR & $\mathrm{HB}$ & Prostate Cancer & 81 & 3 & 0 & 98 & 2 & 0 & Y \\
\hline rs2240307 & Gunes et al. & 2010 & Asian & PCR & $\mathrm{HB}$ & Astrocytoma & 93 & 7 & 0 & 95 & 5 & 0 & Y \\
\hline rs2240307 & Filho et al. & 2011 & Caucasion & TaqMan & $\mathrm{HB}$ & Oral Cancer & $\begin{array}{l}\mathrm{PA}= \\
182\end{array}$ & $\begin{array}{l}\mathrm{PB}= \\
194\end{array}$ & $\mathrm{HA}=2$ & 212 & $\mathrm{HB}=2$ & 238 & NA \\
\hline rs2240307 & Han et al. & 2016 & Asian & $P C R$ & PB & Lung Cancer & 63 & 27 & 12 & 79 & 36 & 5 & Y \\
\hline rs2240307 & Bahl et al. & 2017 & Asian & PCR-RFLP & PB & Lung Cancer & 342 & 34 & 0 & 289 & 16 & 0 & Y \\
\hline rs 2240308 & Kanzaki et al. & 2006 & Asian & PCR-RFLP & PB & $\begin{array}{l}\text { Colorectal } \\
\text { Cancer }\end{array}$ & 54 & 44 & 15 & 42 & 52 & 15 & Y \\
\hline rs2240308 & Kanzaki et al. & 2006 & Asian & PCR-RFLP & PB & $\begin{array}{l}\text { Head and neck } \\
\text { Cancer }\end{array}$ & 25 & 29 & 9 & 42 & 52 & 15 & Y \\
\hline rs2240308 & Kanzaki et al. & 2006 & Asian & PCR-RFLP & PB & Lung Cancer & 81 & 71 & 8 & 42 & 52 & 15 & Y \\
\hline rs2240308 & Gunes et al. & 2009 & Asian & PCR & PB & Lung Cancer & 45 & 47 & 8 & 32 & 52 & 16 & Y \\
\hline rs 2240308 & Gunes et al. & 2010 & Asian & PCR & $\mathrm{HB}$ & Astrocytoma & 39 & 45 & 16 & 32 & 52 & 16 & Y \\
\hline rs2240308 & $\begin{array}{l}\text { Ferna'ndez- } \\
\text { Rozadilla et al. }\end{array}$ & 2010 & Caucasion & MassARRAY & $\mathrm{HB}$ & $\begin{array}{l}\text { Colorectal } \\
\text { Cancer }\end{array}$ & 252 & 423 & 168 & 290 & 442 & 152 & Y \\
\hline rs 2240308 & Pinarbasi et al. & 2010 & Asian & $P C R$ & $\mathrm{HB}$ & Prostate Cancer & 30 & 35 & 19 & 34 & 48 & 18 & Y \\
\hline rs2240308 & $\begin{array}{l}\text { Naghibalhossaini } \\
\text { et al. }\end{array}$ & 2011 & Asian & PCR-RFLP & PB & $\begin{array}{l}\text { Colorectal } \\
\text { Cancer }\end{array}$ & 34 & 57 & 19 & 55 & 98 & 26 & Y \\
\hline rs2240308 & Filho et al. & 2011 & Caucasion & TaqMan & $\mathrm{HB}$ & Oral Cancer & $\begin{array}{l}\mathrm{PA}= \\
196\end{array}$ & $\begin{array}{l}\mathrm{PB}= \\
180\end{array}$ & $\mathrm{HA}=2$ & 226 & $\mathrm{HB}=2$ & 226 & NA \\
\hline rs 2240308 & Mostowska et al. & 2013 & Caucasion & PCR-RFLP & $\mathrm{HB}$ & Ovarian Cancer & 67 & 115 & 46 & 71 & 146 & 65 & Y \\
\hline rs2240308 & Liu et al. & 2014 & Asian & $P C R$ & PB & Lung Cancer & 235 & 216 & 47 & 211 & 255 & 67 & Y \\
\hline rs2240308 & Ma et al. & 2014 & Asian & PCR & $\mathrm{HB}$ & Prostate Cancer & 61 & 31 & 11 & 39 & 52 & 9 & Y \\
\hline rs 2240308 & $\begin{array}{l}\text { Aristizabal- } \\
\text { Pachon et al. }\end{array}$ & 2015 & Caucasion & PCR-RFLP & $\mathrm{PB}$ & Breast Cancer & 20 & 58 & 24 & 44 & 55 & 3 & $\mathrm{~N}$ \\
\hline rs 2240308 & Yadav et al. & 2015 & Asian & PCR-RFLP & PB & $\begin{array}{l}\text { Gallbladder } \\
\text { Cancer }\end{array}$ & 98 & 108 & 44 & 192 & 253 & 119 & N \\
\hline rs2240308 & $\begin{array}{l}\text { Rosales-Reynoso } \\
\text { et al. }\end{array}$ & 2016 & Caucasion & PCR-RFLP & $\mathrm{PB}$ & $\begin{array}{l}\text { Colorectal } \\
\text { Cancer }\end{array}$ & 25 & 109 & 54 & 22 & 59 & 18 & Y \\
\hline rs2240308 & Kim et al. & 2016 & Asian & GoldenGate & $\mathrm{HB}$ & $\begin{array}{l}\text { Hepatocellular } \\
\text { Carcinoma }\end{array}$ & 124 & 100 & 18 & 246 & 195 & 41 & Y \\
\hline rs 2240308 & Han et al. & 2016 & Asian & $P C R$ & $\mathrm{~PB}$ & Lung Cancer & 50 & 34 & 18 & 67 & 43 & 10 & Y \\
\hline rs2240308 & Kim et al. & 2016 & Asian & $\begin{array}{l}\text { Dynamic } 96.96 \\
\text { ArrayTM Assay }\end{array}$ & PB & Lung Cancer & 169 & 142 & 47 & 562 & 436 & 124 & $\mathrm{~N}$ \\
\hline rs2240308 & Liu et al. & 2016 & Asian & MassARRAY & $\mathrm{HB}$ & $\begin{array}{l}\text { Papillary Thyroid } \\
\text { Carcinoma }\end{array}$ & 27 & 24 & 2 & 17 & 29 & 4 & Y \\
\hline
\end{tabular}


Table 1 Characteristics of the enrolled studies on AXIN2 Polymorphism and cancer (Continued)

\begin{tabular}{|c|c|c|c|c|c|c|c|c|c|c|c|c|c|}
\hline \multirow[t]{2}{*}{ Polymorphism } & \multirow[t]{2}{*}{ First author } & \multirow[t]{2}{*}{ Year } & \multirow[t]{2}{*}{ Ethnicity } & \multirow{2}{*}{$\begin{array}{l}\text { Genotyping } \\
\text { Method }\end{array}$} & \multirow{2}{*}{$\begin{array}{l}\text { Source } \\
\text { of } \\
\text { Control }\end{array}$} & \multirow[t]{2}{*}{ Cancer Type } & \multicolumn{3}{|c|}{ Cases } & \multicolumn{4}{|c|}{ Controls } \\
\hline & & & & & & & PAA & PAB & PBB & HAA & HAB & $\mathrm{HBB}$ & HWE \\
\hline rs2240308 & Bahl et al. & 2017 & Asian & PCR-RFLP & PB & Lung Cancer & 99 & 150 & 54 & 81 & 144 & 80 & Y \\
\hline rs35285779 & Gunes et al. & 2009 & Asian & $P C R$ & PB & Lung Cancer & 77 & 20 & 3 & 64 & 28 & 8 & Y \\
\hline rs35285779 & Pinarbasi et al. & 2010 & Asian & $P C R$ & $\mathrm{HB}$ & Prostate Cancer & 69 & 15 & 0 & 61 & 32 & 7 & Y \\
\hline rs35285779 & Gunes et al. & 2010 & Asian & $P C R$ & $\mathrm{HB}$ & Astrocytoma & 70 & 25 & 5 & 64 & 28 & 8 & Y \\
\hline rs35285779 & Bahl et al. & 2017 & Asian & PCR-RFLP & PB & Lung Cancer & 255 & 46 & 2 & 248 & 55 & 2 & Y \\
\hline rs35415678 & Gunes et al. & 2009 & Asian & $P C R$ & PB & Lung Cancer & 91 & 9 & 0 & 86 & 14 & 0 & Y \\
\hline rs35415678 & Pinarbasi et al. & 2010 & Asian & $P C R$ & $\mathrm{HB}$ & Prostate Cancer & 83 & 1 & 0 & 99 & 1 & 0 & Y \\
\hline rs35415678 & Gunes et al. & 2010 & Asian & $P C R$ & $\mathrm{HB}$ & Astrocytoma & 87 & 13 & 0 & 86 & 14 & 0 & Y \\
\hline rs35415678 & Bahl et al. & 2017 & Asian & PCR-RFLP & PB & Lung Cancer & 257 & 46 & 0 & 261 & 44 & 0 & Y \\
\hline rs3923086 & Wang et al. & 2008 & Caucasion & GoldenGate & PB & Breast Cancer & 238 & 395 & 164 & 284 & 419 & 139 & Y \\
\hline rs3923086 & Filho et al. & 2011 & Caucasion & TaqMan & $\mathrm{HB}$ & Oral Cancer & $\begin{array}{l}\mathrm{PA}= \\
172\end{array}$ & $\begin{array}{l}P B= \\
204\end{array}$ & $H A=$ & 212 & $H B=$ & 238 & NA \\
\hline rs3923086 & Alanazi et al. & 2013 & Asian & TaqMan & PB & Breast Cancer & 27 & 41 & 31 & 16 & 42 & 35 & Y \\
\hline rs3923086 & Liu et al. & 2016 & Asian & MassARRAY & $\mathrm{HB}$ & $\begin{array}{l}\text { Papillary Thyroid } \\
\text { Carcinoma }\end{array}$ & 47 & 8 & 0 & 34 & 15 & 1 & Y \\
\hline rs3923086 & Parine et al. & 2019 & Asian & TaqMan & PB & $\begin{array}{l}\text { Colorectal } \\
\text { Cancer }\end{array}$ & 48 & 52 & 21 & 41 & 50 & 19 & Y \\
\hline rs3923087 & Wang et al. & 2008 & Caucasion & GoldenGate & PB & Breast Cancer & 47 & 292 & 458 & 39 & 278 & 525 & Y \\
\hline rs3923087 & Filho et al. & 2011 & Caucasion & TaqMan & $\mathrm{HB}$ & Oral Cancer & $\begin{array}{l}\mathrm{PA}= \\
70\end{array}$ & $\begin{array}{l}P B= \\
306\end{array}$ & $\mathrm{HA}=$ & 130 & $\mathrm{HB}=$ & 320 & NA \\
\hline rs3923087 & Mostowska et al. & 2013 & Caucasion & PCR-RFLP & $\mathrm{HB}$ & Ovarian Cancer & 10 & 84 & 133 & 14 & 97 & 171 & Y \\
\hline rs3923087 & Alanazi et al. & 2013 & Asian & TaqMan & PB & Breast Cancer & 45 & 35 & 18 & 24 & 50 & 19 & Y \\
\hline rs3923087 & Parine et al. & 2019 & Asian & TaqMan & PB & $\begin{array}{l}\text { Colorectal } \\
\text { Cancer }\end{array}$ & 35 & 56 & 32 & 37 & 50 & 23 & Y \\
\hline rs4072245 & Gunes et al. & 2009 & Asian & $P C R$ & PB & Lung Cancer & 73 & 27 & 0 & 80 & 20 & 0 & Y \\
\hline rs4072245 & Pinarbasi et al. & 2010 & Asian & $P C R$ & $\mathrm{HB}$ & Prostate Cancer & 73 & 11 & 0 & 78 & 22 & 0 & Y \\
\hline rs4072245 & Gunes et al. & 2010 & Asian & $P C R$ & $\mathrm{HB}$ & Astrocytoma & 82 & 18 & 0 & 80 & 20 & 0 & Y \\
\hline rs4791171 & Wang et al. & 2008 & Caucasion & GoldenGate & PB & Breast Cancer & 83 & 332 & 383 & 61 & 349 & 433 & Y \\
\hline rs4791171 & Filho et al. & 2011 & Caucasion & TaqMan & $\mathrm{HB}$ & Oral Cancer & $\begin{array}{l}P A= \\
124\end{array}$ & $\begin{array}{l}P B= \\
252\end{array}$ & $\mathrm{HA}=$ & 136 & $H B=$ & 316 & NA \\
\hline rs4791171 & Alanazi et al. & 2013 & Asian & TaqMan & PB & Breast Cancer & 34 & 44 & 21 & 22 & 44 & 17 & Y \\
\hline rs4791171 & Yadav et al. & 2015 & Asian & PCR-RFLP & PB & $\begin{array}{l}\text { Gallbladder } \\
\text { Cancer }\end{array}$ & 35 & 118 & 97 & 88 & 248 & 228 & Y \\
\hline rs4791171 & Parine et al. & 2019 & Asian & TaqMan & PB & $\begin{array}{l}\text { Colorectal } \\
\text { Cancer }\end{array}$ & 40 & 55 & 27 & 38 & 48 & 24 & Y \\
\hline rs7219582 & Gunes et al. & 2009 & Asian & $P C R$ & PB & Lung Cancer & 97 & 3 & 0 & 96 & 4 & 0 & Y \\
\hline rs7219582 & Pinarbasi et al. & 2010 & Asian & $P C R$ & $\mathrm{HB}$ & Prostate Cancer & 81 & 3 & 0 & 95 & 5 & 0 & Y \\
\hline rs7219582 & Gunes et al. & 2010 & Asian & PCR & $\mathrm{HB}$ & Astrocytoma & 91 & 9 & 0 & 96 & 4 & 0 & Y \\
\hline rs7219582 & Bahl et al. & 2017 & Asian & PCR-RFLP & PB & Lung Cancer & 87 & 205 & 11 & 42 & 263 & 0 & N \\
\hline rs7224837 & Filho et al. & 2011 & Caucasion & TaqMan & $\mathrm{HB}$ & Oral Cancer & 342 & 34 & & 400 & 50 & & NA \\
\hline rs7224837 & Mostowska et al. & 2013 & Caucasion & PCR-RFLP & $\mathrm{HB}$ & Ovarian Cancer & 161 & 61 & 6 & 203 & 71 & 8 & Y \\
\hline rs7224837 & Jeanne et al. & 2015 & Caucasion & $\begin{array}{l}\text { iSelect } \\
\text { genotyping array }\end{array}$ & $\mathrm{HB}$ & Bladder Cancer & 646 & 151 & 6 & 616 & 169 & 17 & Y \\
\hline rs9915936 & Gunes et al. & 2009 & Asian & $P C R$ & PB & Lung Cancer & 91 & 9 & 0 & 88 & 12 & 0 & Y \\
\hline
\end{tabular}


Table 1 Characteristics of the enrolled studies on AXIN2 Polymorphism and cancer (Continued)

\begin{tabular}{|c|c|c|c|c|c|c|c|c|c|c|c|c|c|}
\hline \multirow[t]{2}{*}{ Polymorphism } & \multirow[t]{2}{*}{ First author } & \multirow[t]{2}{*}{ Year } & \multirow[t]{2}{*}{ Ethnicity } & \multirow{2}{*}{$\begin{array}{l}\text { Genotyping } \\
\text { Method }\end{array}$} & \multirow{2}{*}{$\begin{array}{l}\text { Source } \\
\text { of } \\
\text { Control }\end{array}$} & \multirow[t]{2}{*}{ Cancer Type } & \multicolumn{3}{|c|}{ Cases } & \multicolumn{4}{|c|}{ Controls } \\
\hline & & & & & & & $\overline{\text { PAA }}$ & PAB & $\overline{\text { PBB }}$ & HAA & HAB & HBB & $\overline{\text { HWE }}$ \\
\hline rs9915936 & Pinarbasi et al. & 2010 & Asian & $P C R$ & $\mathrm{HB}$ & Prostate Cancer & 77 & 7 & 0 & 92 & 8 & 0 & $\mathrm{Y}$ \\
\hline rs9915936 & Gunes et al. & 2010 & Asian & $P C R$ & $\mathrm{HB}$ & Astrocytoma & 91 & 9 & 0 & 88 & 12 & 0 & Y \\
\hline rs9915936 & Bahl et al. & 2017 & Asian & PCR-RFLP & $\mathrm{PB}$ & Lung Cancer & 268 & 29 & 6 & 249 & 51 & 5 & Y \\
\hline
\end{tabular}

$H B$ Hospital Based, PB Population Based, HWE Hardy Weinberg Equilibrium, $Y$ polymorphisms conformed to HWE in the control group, $N$ polymorphisms didn't conform to HWE in the control group, NA not available

[25, 26, 34, 43, 45], four studies focused on $r s 7219582$ [16, 28-30], three studies focused on rs 7224837 [34, 41, 46], four studies focused on rs9915936 [16, 28-30]. Table 1 showed all details of the involved studies. Newcastle-Ottawa Scale (NOS) [40] was performed to assess the quality of each included study, and the results were showed in Table S1.

\section{AXIN-2 polymorphism and risk of cancers}

Thirteen polymorphisms of $A X I N-2$ were analyzed in the study. For $r s 11079571$ polymorphism, two studies were related to breast cancer and another was involved in acute leukemia. Among which, two were about Asian population and one was based on Caucasian. The sources of all three controls were population based. All of the three genotype distributions of controls of rs11079571 studies were conformed to HWE, For the rs1133683 polymorphism, six studies met the criteria, including two lung cancers and one prostate cancer, astrocytoma, ovarian cancer and colorectal cancer, respectively. Among them, five studies related to Asian and one study concerned about Caucasion population. As to $r s 2240307$ polymorphism, six studies were involved, three of them were about lung cancer, and the other three were about oral cancer, prostate cancer, astrocytoma, respectively. For the $r s 2240308$ polymorphism, 20 studies were connected, among which, six were about lung cancer, four were about colorectal cancer, two were about prostate cancer, and another eight were about head and neck cancer, astrocytoma, oral cancer, ovarian cancer, breast cancer, gallbladder cancer, papillary thyroid carcinoma and hepatocellular carcinoma, respectively. Fifteen studies were Asian population based and five were Caucasion based. For rs35285779 polymorphism, two studies were about lung cancer, another two were about prostate cancer and astrocytoma, respectively. All the four studies were Asian population based. For rs35415678 polymorphism, two studies were connected to lung cancer and another two were about prostate cancer and astrocytoma, respectively. For $r s 3923086$ polymorphism, five studies were involved, two of which were about breast cancer and another three were oral cancer, papillary thyroid carcinoma and colorectal cancer, respectively. For rs3923087 polymorphism, five studies were involved, two of which were about breast cancer and another three were oral cancer, ovarian cancer and colorectal cancer, respectively. For rs4072245 polymorphism, there studies were about lung cancer, prostate cancer and astrocytoma, respectively. For $r s 4791171$ polymorphism, five studies were involved, two of which were about breast cancer and another three were colorectal cancer, oral cancer and gallbladder cancer, respectively. As to rs7219582 polymorphism, four studies were included, two of which were about lung cancer, and another two were prostate cancer and astrocytoma, respectively. For rs7224837 polymorphism, three studies were about oral cancer, ovarian cancer and bladder cancer, respectively. As to rs 9915936 polymorphism, four studies were included, two of which were focused on lung cancer, and another two were about prostate cancer and astrocytoma, respectively.

Table 2 and Table S2 showed the results about AXIN-2 polymorphisms and cancer susceptibility. There were significant associations in four genetic models between rs11079571 polymorphism and overall cancer risk, including allelic contrast model (B vs. A: $\mathrm{OR}=0.539,95 \% \mathrm{CI}=$ 0.478-0.609, PAdjust $=0.025$ ), homozygote comparison model (BB vs. AA: OR $=0.22,95 \% \mathrm{CI}=0.164-0.295$, PAdjust $<0.001$ ), heterozygote comparison model (BA vs. AA: $\mathrm{OR}=0.292,95 \% \mathrm{CI}=0.216-0.394$, PAdjust $<0.001)$ and dominant comparison model $(\mathrm{BB}+\mathrm{BA}$ vs. $\mathrm{AA}: \mathrm{OR}=$ $0.249,95 \% \mathrm{CI}=0.189-0.33$, PAdjust $<0.001$ ), whereas, there was no significant association in recessive comparison model (BB vs. $\mathrm{BA}+\mathrm{AA}$ : $\mathrm{OR}=0.619,95 \% \mathrm{CI}=0.531-$ 0.723 , PAdjust $=0.11$ ). What's more, the stratification analysis of ethnicity also reflected $r s 11079571$ polymorphism risk to cancers in Asian population in $\mathrm{B}$ vs. $\mathrm{A}, \mathrm{BB}$ vs. AA, $\mathrm{BA}$ vs. $\mathrm{AA}$ and $\mathrm{BB}+\mathrm{BA}$ vs. $\mathrm{AA}$ models (PAdjust<0.05). For cancer type analysis, rs11079571 polymorphism showed strong association with risk of breast cancer in BA vs. AA and BB + BA vs. AA models (PAdjust $<0.05$ ) (Table 2, Figure S1). For $r s 1133683$, which had significant association with overall cancer risk in $\mathrm{BB}$ vs. $\mathrm{AA}$ and $\mathrm{BB}$ vs. $\mathrm{BA}+\mathrm{AA}$ models (PAdjust $<0.05$ ), and with Asian population in $\mathrm{BB}$ vs. BA+AA model (PAdjust $<0.05$ ), with population based $(\mathrm{PB})$ source of control in $\mathrm{BB}$ vs. $\mathrm{AA}$ and $\mathrm{BB}$ vs. BA+AA models (PAdjust< 0.05 ) (Table 2, Figure S2). For $r$ 2240308, which showed significant correlation with risk of Asian population in $\mathrm{BA}$ vs. $\mathrm{AA}$ and $\mathrm{BB}+\mathrm{BA}$ vs. AA models (PAdjust<0.05) (Table 2, Fig. 2). For 
Table 2 Results of pooled analysis for AXIN2 Polymorphism and cancer susceptibility

\begin{tabular}{|c|c|c|c|c|c|c|c|c|}
\hline Polymorphism & Comparison & Subgroup & $\mathrm{N}$ & $\mathbf{P}_{\mathrm{H}}$ & $P_{z}$ & $P_{\text {Adjust }}$ & OR \& 95\%Cl (Random) & OR \& 95\%Cl (Fixed) \\
\hline rs11079571 & B vs. A & Overall & 3 & $<0.001$ & 0.005 & $0.025^{*}$ & $0.459(0.266-0.794)$ & $0.539(0.478-0.609)$ \\
\hline rs11079571 & BB vs. AA & Overall & 3 & 0.001 & $<0.001$ & $<0.001^{*}$ & $0.2(0.085-0.469)$ & $0.22(0.164-0.295)$ \\
\hline rs11079571 & BA vs. AA & Overall & 3 & 0.081 & $<0.001$ & $<0.001^{*}$ & $0.322(0.192-0.54)$ & $0.292(0.216-0.394)$ \\
\hline rs11079571 & $\mathrm{BB}+\mathrm{BA}$ vs. $\mathrm{AA}$ & Overall & 3 & 0.08 & $<0.001$ & $<0.001^{*}$ & $0.265(0.162-0.433)$ & $0.249(0.189-0.33)$ \\
\hline rs11079571 & $B B$ vs. $B A+A A$ & Overall & 3 & $<0.001$ & 0.022 & 0.11 & $0.436(0.215-0.887)$ & $0.619(0.531-0.723)$ \\
\hline rs11079571 & B vs. A & Asian & 2 & 0.002 & 0.001 & $0.005^{*}$ & $0.351(0.191-0.646)$ & $0.407(0.345-0.479)$ \\
\hline rs11079571 & $B B$ vs. $A A$ & Asian & 2 & 0.007 & $<0.001$ & $<0.001^{*}$ & $0.135(0.045-0.407)$ & $0.178(0.127-0.251)$ \\
\hline rs11079571 & BA vs. AA & Asian & 2 & 0.416 & $<0.001$ & $<0.001^{*}$ & $0.246(0.174-0.346)$ & $0.247(0.175-0.348)$ \\
\hline rs11079571 & $\mathrm{BB}+\mathrm{BA}$ vs. $\mathrm{AA}$ & Asian & 2 & 0.575 & $<0.001$ & $<0.001^{*}$ & $0.216(0.157-0.297)$ & $0.215(0.156-0.295)$ \\
\hline rs11079571 & $B B$ vs. $B A+A A$ & Asian & 2 & $<0.001$ & 0.083 & 0.415 & $0.311(0.083-1.166)$ & $0.463(0.368-0.582)$ \\
\hline rs11079571 & B vs. A & Breast Cancer & 2 & $<0.001$ & 0.148 & 0.74 & $0.446(0.150-1.332)$ & $0.594(0.507-0.697)$ \\
\hline rs11079571 & $B B$ vs. $A A$ & Breast Cancer & 2 & $<0.001$ & 0.056 & 0.28 & $0.182(0.032-1.047)$ & $0.209(0.133-0.329)$ \\
\hline rs11079571 & BA vs. AA & Breast Cancer & 2 & 0.289 & $<0.001$ & $<0.001^{*}$ & $0.419(0.255-0.689)$ & $0.416(0.261-0.662)$ \\
\hline rs11079571 & $\mathrm{BB}+\mathrm{BA}$ vs. $\mathrm{AA}$ & Breast Cancer & 2 & 0.041 & 0.009 & $0.045^{*}$ & $0.294(0.118-0.734)$ & $0.285(0.184-0.441)$ \\
\hline rs11079571 & $B B$ vs. $B A+A A$ & Breast Cancer & 2 & $<0.001$ & 0.202 & 1 & $0.356(0.073-1.74)$ & $0.63(0.52-0.764)$ \\
\hline rs1133683 & B vs. A & Overall & 6 & $<0.001$ & 0.664 & 1.000 & $1.076(0.773-1.498)$ & $1.14(1.021-1.273)$ \\
\hline rs1133683 & BB vs. AA & Overall & 6 & $<0.001$ & 0.005 & $0.025^{*}$ & $0.258(0.101-0.657)$ & $0.391(0.284-0.539)$ \\
\hline rs1133683 & BA vs. AA & Overall & 6 & $<0.001$ & 0.036 & 0.18 & $2.079(1.048-4.126)$ & $2.298(1.948-2.71)$ \\
\hline rs1133683 & $\mathrm{BB}+\mathrm{BA}$ vs. $\mathrm{AA}$ & Overall & 6 & $<0.001$ & 0.1 & 0.5 & $1.78(0.895-3.538)$ & $1.962(1.673-2.301)$ \\
\hline rs1133683 & $B B$ vs. $B A+A A$ & Overall & 6 & $<0.001$ & $<0.001$ & $<0.001^{*}$ & $0.162(0.08-0.328)$ & $0.206(0.152-0.278)$ \\
\hline rs1133683 & B vs. A & Asian & 5 & $<0.001$ & 0.2 & 1.000 & $1.212(0.904-1.625)$ & $1.25(1.11-1.408)$ \\
\hline rs1133683 & $B B$ vs. $A A$ & Asian & 5 & $<0.001$ & 0.026 & 0.13 & $0.283(0.093-0.858)$ & $0.469(0.329-0.67)$ \\
\hline rs1133683 & BA vs. AA & Asian & 5 & $<0.001$ & 0.01 & 0.05 & $2.51(1.247-5.052)$ & $2.627(2.203-3.132)$ \\
\hline rs1133683 & $B B+B A$ vs. $A A$ & Asian & 5 & $<0.001$ & 0.025 & 0.125 & $2.186(1.105-4.322)$ & $2.283(1.926-2.707)$ \\
\hline rs1133683 & $B B$ vs. $B A+A A$ & Asian & 5 & $<0.001$ & $<0.001$ & $<0.001^{*}$ & $0.154(0.062-0.383)$ & $0.21(0.15-0.295)$ \\
\hline rs1133683 & B vs. A & PB & 4 & $<0.001$ & 0.828 & 1.000 & $1.051(0.67-1.651)$ & $1.146(1.01-1.302)$ \\
\hline rs1133683 & BB vs. AA & PB & 4 & 0.006 & 0.001 & $0.005^{*}$ & $0.256(0.113-0.584)$ & $0.349(0.241-0.504)$ \\
\hline rs1133683 & BA vs. AA & PB & 4 & $<0.001$ & 0.118 & 0.59 & $2.112(0.827-5.395)$ & $2.541(2.093-3.084)$ \\
\hline rs1133683 & $B B+B A$ vs. $A A$ & PB & 4 & $<0.001$ & 0.23 & 1.000 & $1.773(0.696-4.515)$ & $2.142(1.777-2.582)$ \\
\hline rs1133683 & $B B$ vs. $B A+A A$ & PB & 4 & 0.045 & $<0.001$ & $<0.001^{*}$ & $0.16(0.086-0.297)$ & $0.184(0.13-0.259)$ \\
\hline rs1133683 & B vs. A & $H B$ & 2 & 0.004 & 0.72 & 1.000 & $1.127(0.587-2.163)$ & $1.12(0.895-1.401)$ \\
\hline rs1133683 & $B B$ vs. $A A$ & $\mathrm{HB}$ & 2 & $<0.001$ & 0.46 & 1.000 & $0.265(0.008-8.979)$ & $0.556(0.291-1.062)$ \\
\hline rs1133683 & BA vs. AA & $H B$ & 2 & $<0.001$ & 0.249 & 1.000 & $2.001(0.615-6.508)$ & $1.788(1.305-2.45)$ \\
\hline rs1133683 & $\mathrm{BB}+\mathrm{BA}$ vs. $\mathrm{AA}$ & $\mathrm{HB}$ & 2 & $<0.001$ & 0.361 & 1.000 & $1.782(0.515-6.161)$ & $1.572(1.159-2.132)$ \\
\hline rs1133683 & $B B$ vs. $B A+A A$ & $H B$ & 2 & $<0.001$ & 0.186 & 0.93 & $0.166(0.012-2.381)$ & $0.297(0.158-0.559)$ \\
\hline rs1133683 & B vs. A & Lung Cancer & 2 & 0.016 & 0.767 & 1.000 & $1.071(0.68-1.687)$ & $1.196(1.023-1.399)$ \\
\hline rs1133683 & BB vs. AA & Lung Cancer & 2 & 0.228 & 0.008 & $0.04^{*}$ & $0.491(0.263-0.918)$ & $0.53(0.333-0.845)$ \\
\hline rs1133683 & BA vs. AA & Lung Cancer & 2 & $<0.001$ & 0.317 & 1.000 & $2.143(0.482-9.522)$ & $2.695(2.109-3.442)$ \\
\hline rs1133683 & $\mathrm{BB}+\mathrm{BA}$ vs. $\mathrm{AA}$ & Lung Cancer & 2 & $<0.001$ & 0.387 & 1.000 & $1.888(0.448-7.959)$ & $2.36(1.86-2.995)$ \\
\hline rs1133683 & $B B$ vs. $B A+A A$ & Lung Cancer & 2 & 0.39 & $<0.001$ & $<0.001^{*}$ & $0.21(0.136-0.325)$ & $0.212(0.138-0.328)$ \\
\hline rs1133683 & B vs. A & Y & 5 & $<0.001$ & 0.898 & 1.000 & $1.028(0.673-1.571)$ & $1.036(0.899-1.193)$ \\
\hline rs1133683 & BB vs. AA & Y & 5 & $<0.001$ & 0.008 & $0.04^{*}$ & $0.211(0.067-0.666)$ & $0.293(0.195-0.44)$ \\
\hline rs1133683 & BA vs. AA & Y & 5 & $<0.001$ & 0.14 & 0.7 & $1.767(0.83-3.762)$ & $1.753(1.434-2.142)$ \\
\hline rs1133683 & $\mathrm{BB}+\mathrm{BA}$ vs. $\mathrm{AA}$ & Y & 5 & $<0.001$ & 0.287 & 1.000 & $1.512(0.706-3.241)$ & $1.499(1.236-1.818)$ \\
\hline
\end{tabular}


Table 2 Results of pooled analysis for AXIN2 Polymorphism and cancer susceptibility (Continued)

\begin{tabular}{|c|c|c|c|c|c|c|c|c|}
\hline Polymorphism & Comparison & Subgroup & $\mathrm{N}$ & $P_{H}$ & $P_{z}$ & $P_{\text {Adjust }}$ & OR \& 95\%Cl (Random) & OR \& 95\%Cl (Fixed) \\
\hline rs1133683 & $B B$ vs. $B A+A A$ & Y & 5 & $<0.001$ & $<0.001$ & $<0.001^{*}$ & $0.152(0.057-0.405)$ & $0.215(0.146-0.315)$ \\
\hline rs2240308 & B vs. A & Overall & 20 & $<0.001$ & 0.402 & 1.000 & $0.949(0.841-1.072)$ & $0.962(0.906-1.02)$ \\
\hline rs2240308 & $B B$ vs. $A A$ & Overall & 19 & $<0.001$ & 0.722 & 1.000 & $0.952(0.726-1.248)$ & $0.966(0.849-1.1)$ \\
\hline rs2240308 & BA vs. AA & Overall & 19 & 0.016 & 0.089 & 0.445 & 0.887(0.773-1.018) & $0.915(0.834-1.004)$ \\
\hline rs2240308 & $\mathrm{BB}+\mathrm{BA}$ vs. $\mathrm{AA}$ & Overall & 19 & $<0.001$ & 0.176 & 0.88 & 0.895(0.763-1.051) & $0.923(0.846-1.007)$ \\
\hline rs2240308 & $B B$ vs. $B A+A A$ & Overall & 19 & $<0.001$ & 0.963 & 1.000 & $1.005(0.811-1.246)$ & $1.006(0.895-1.13)$ \\
\hline rs2240308 & B vs. A & Asian & 15 & 0.01 & 0.017 & 0.085 & $0.867(0.772-0.974)$ & $0.879(0.815-0.947)$ \\
\hline rs2240308 & BB vs. AA & Asian & 15 & 0.019 & 0.072 & 0.36 & $0.799(0.626-1.021)$ & $0.806(0.686-0.946)$ \\
\hline rs2240308 & BA vs. AA & Asian & 15 & 0.268 & 0.002 & $0.01^{*}$ & $0.828(0.731-0.939)$ & $0.84(0.753-0.937)$ \\
\hline rs2240308 & $\mathrm{BB}+\mathrm{BA}$ vs. $\mathrm{AA}$ & Asian & 15 & 0.066 & 0.004 & $0.02^{*}$ & $0.811(0.704-0.934)$ & $0.835(0.754-0.926)$ \\
\hline rs2240308 & $B B$ vs. $B A+A A$ & Asian & 15 & 0.053 & 0.273 & 1.000 & $0.889(0.721-1.097)$ & $0.874(0.754-1.013)$ \\
\hline rs2240308 & B vs. A & Caucasian & 5 & $<0.001$ & 0.138 & 0.69 & $1.228(0.936-1.61)$ & $1.119(1.016-1.233)$ \\
\hline rs2240308 & BB vs. $A A$ & Caucasian & 4 & $<0.001$ & 0.082 & 0.41 & $2.069(0.912-4.692)$ & $1.375(1.101-1.716)$ \\
\hline rs2240308 & BA vs. AA & Caucasian & 4 & 0.044 & 0.224 & 1.000 & $1.253(0.871-1.801)$ & $1.141(0.957-1.36)$ \\
\hline rs2240308 & $B B+B A$ vs. $A A$ & Caucasian & 4 & 0.002 & 0.143 & 0.715 & $1.421(0.888-2.274)$ & $1.198(1.014-1.414)$ \\
\hline rs2240308 & $B B$ vs. $B A+A A$ & Caucasian & 4 & 0.001 & 0.112 & 0.56 & $1.6(0.896-2.858)$ & $1.277(1.053-1.548)$ \\
\hline rs2240308 & B vs. A & PB & 12 & $<0.001$ & 0.894 & 1.000 & $0.987(0.815-1.195)$ & $0.944(0.871-1.022)$ \\
\hline rs2240308 & BB vs. AA & PB & 12 & $<0.001$ & 0.955 & 1.000 & $1.012(0.67-1.529)$ & $0.919(0.777-1.087)$ \\
\hline rs2240308 & BA vs. AA & PB & 12 & 0.064 & 0.364 & 1.000 & 0.924(0.78-1.096) & $0.913(0.81-1.028)$ \\
\hline rs2240308 & $B B+B A$ vs. $A A$ & PB & 12 & $<0.001$ & 0.644 & 1.000 & $0.949(0.762-1.183)$ & $0.911(0.814-1.019)$ \\
\hline rs2240308 & $B B$ vs. $B A+A A$ & PB & 12 & $<0.001$ & 0.893 & 1.000 & $1.023(0.734-1.425)$ & $0.96(0.823-1.119)$ \\
\hline rs2240308 & B vs. A & $\mathrm{HB}$ & 8 & 0.114 & 0.719 & 1.000 & $0.935(0.822-1.064)$ & $0.984(0.901-1.075)$ \\
\hline rs2240308 & $B B$ vs. $A A$ & $H B$ & 7 & 0.376 & 0.705 & 1.000 & $1.015(0.812-1.27)$ & $1.04(0.849-1.273)$ \\
\hline rs2240308 & BA vs. AA & $\mathrm{HB}$ & 7 & 0.047 & 0.1 & 0.5 & $0.839(0.681-1.034)$ & $0.919(0.808-1.045)$ \\
\hline rs2240308 & $\mathrm{BB}+\mathrm{BA}$ vs. $\mathrm{AA}$ & $H B$ & 7 & 0.043 & 0.128 & 0.64 & 0.855(0.699-1.046) & $0.937(0.828-1.06)$ \\
\hline rs2240308 & $B B$ vs. $B A+A A$ & $\mathrm{HB}$ & 7 & 0.684 & 0.444 & 1.000 & $1.075(0.897-1.287)$ & $1.073(0.896-1.284)$ \\
\hline rs2240308 & B vs. A & Colorectal Cancer & 4 & 0.15 & 0.056 & 0.28 & $1.108(0.918-1.336)$ & $1.116(0.997-1.249)$ \\
\hline rs2240308 & $B B$ vs. $A A$ & Colorectal Cancer & 4 & 0.192 & 0.031 & 0.155 & 1.314(0.903-1.911) & $1.295(1.024-1.637)$ \\
\hline rs2240308 & BA vs. AA & Colorectal Cancer & 4 & 0.2 & 0.548 & 1.000 & $1.031(0.779-1.363)$ & $1.057(0.882-1.266)$ \\
\hline rs2240308 & $B B+B A$ vs. $A A$ & Colorectal Cancer & 4 & 0.113 & 0.252 & 1.000 & 1.083(0.794-1.478) & $1.105(0.931-1.312)$ \\
\hline rs2240308 & $B B$ vs. $B A+A A$ & Colorectal Cancer & 4 & 0.563 & 0.036 & 0.18 & $1.241(1.011-1.524)$ & $1.245(1.015-1.527)$ \\
\hline rs2240308 & B vs. A & Prostate Cancer & 2 & 0.099 & 0.452 & 1.000 & $0.828(0.507-1.353)$ & 0.832(0.619-1.119) \\
\hline rs2240308 & BB vs. AA & Prostate Cancer & 2 & 0.509 & 0.987 & 1.000 & 1.004(0.539-1.869) & $1.005(0.54-1.871)$ \\
\hline rs2240308 & BA vs. AA & Prostate Cancer & 2 & 0.088 & 0.127 & 0.635 & $0.555(0.26-1.183)$ & $0.542(0.35-0.84)$ \\
\hline rs2240308 & $\mathrm{BB}+\mathrm{BA}$ vs. $\mathrm{AA}$ & Prostate Cancer & 2 & 0.078 & 0.219 & 1.000 & $0.633(0.305-1.313)$ & $0.62(0.412-0.934)$ \\
\hline rs2240308 & $B B$ vs. $B A+A A$ & Prostate Cancer & 2 & 0.872 & 0.39 & 1.000 & $1.284(0.726-2.27)$ & $1.284(0.726-2.27)$ \\
\hline rs2240308 & B vs. A & Lung Cancer & 6 & $<0.001$ & 0.176 & 0.88 & $0.854(0.678-1.074)$ & $0.875(0.791-0.967)$ \\
\hline rs2240308 & BB vs. AA & Lung Cancer & 6 & $<0.001$ & 0.199 & 0.995 & $0.714(0.427-1.194)$ & $0.776(0.626-0.962)$ \\
\hline rs2240308 & BA vs. AA & Lung Cancer & 6 & 0.317 & 0.069 & 0.345 & $0.868(0.736-1.023)$ & $0.873(0.755-1.01)$ \\
\hline rs2240308 & $B B+B A$ vs. $A A$ & Lung Cancer & 6 & 0.022 & 0.218 & 1.000 & $0.827(0.648-1.056)$ & $0.857(0.747-0.983)$ \\
\hline rs2240308 & $B B$ vs. $B A+A A$ & Lung Cancer & 6 & 0.002 & 0.272 & 1.000 & $0.784(0.508-1.211)$ & 0.817(0.669-0.998) \\
\hline rs2240308 & B vs. A & Y & 16 & $<0.001$ & 0.099 & 0.495 & $0.899(0.792-1.02)$ & $0.928(0.866-0.994)$ \\
\hline rs2240308 & BB vs. $A A$ & Y & 16 & 0.001 & 0.281 & 1.000 & 0.862(0.659-1.129) & $0.904(0.78-1.048)$ \\
\hline rs2240308 & BA vs. AA & Y & 16 & 0.097 & 0.018 & 0.09 & $0.843(0.732-0.972)$ & $0.874(0.786-0.971)$ \\
\hline
\end{tabular}


Table 2 Results of pooled analysis for AXIN2 Polymorphism and cancer susceptibility (Continued)

\begin{tabular}{|c|c|c|c|c|c|c|c|c|}
\hline Polymorphism & Comparison & Subgroup & $\mathrm{N}$ & $P_{H}$ & $P_{z}$ & $P_{\text {Adjust }}$ & OR \& 95\%Cl (Random) & OR \& 95\%Cl (Fixed) \\
\hline rs2240308 & $\mathrm{BB}+\mathrm{BA}$ vs. $\mathrm{AA}$ & Y & 16 & 0.008 & 0.03 & 0.15 & $0.838(0.714-0.983)$ & $0.875(0.792-0.967)$ \\
\hline rs2240308 & $B B$ vs. $B A+A A$ & Y & 16 & 0.011 & 0.604 & 1.000 & $0.945(0.761-1.172)$ & $0.962(0.843-1.099)$ \\
\hline rs2240308 & B vs. A & $\mathrm{N}$ & 4 & $<0.001$ & 0.347 & 1.000 & $1.174(0.84-1.64)$ & $1.056(0.944-1.182)$ \\
\hline rs2240308 & BB vs. AA & $\mathrm{N}$ & 3 & $<0.001$ & 0.21 & 1.000 & $1.961(0.684-5.618)$ & $1.199(0.922-1.561)$ \\
\hline rs2240308 & BA vs. AA & N & 3 & 0.022 & 0.468 & 1.000 & $1.171(0.765-1.793)$ & $1.066(0.88-1.292)$ \\
\hline rs2240308 & $\mathrm{BB}+\mathrm{BA}$ vs. $\mathrm{AA}$ & N & 3 & 0.001 & 0.347 & 1.000 & $1.304(0.75-2.265)$ & $1.095(0.915-1.31)$ \\
\hline rs2240308 & $B B$ vs. $B A+A A$ & $\mathrm{~N}$ & 3 & $<0.001$ & 0.243 & 1.000 & $1.648(0.713-3.81)$ & $1.176(0.919-1.504)$ \\
\hline rs35285779 & B vs. A & Overall & 4 & 0.068 & 0.011 & 0.055 & 0.603(0.409-0.889) & $0.632(0.496-0.806)$ \\
\hline rs35285779 & $B B$ vs. $A A$ & Overall & 4 & 0.378 & 0.038 & 0.19 & $0.43(0.194-0.955)$ & $0.368(0.176-0.77)$ \\
\hline rs35285779 & BA vs. AA & Overall & 4 & 0.384 & 0.009 & $0.045^{*}$ & $0.685(0.513-0.915)$ & $0.684(0.514-0.909)$ \\
\hline rs35285779 & $B B+B A$ vs. $A A$ & Overall & 4 & 0.155 & 0.001 & $0.005^{*}$ & $0.613(0.421-0.893)$ & $0.639(0.486-0.839)$ \\
\hline rs35285779 & $B B$ vs. $B A+A A$ & Overall & 4 & 0.448 & 0.017 & 0.085 & $0.473(0.219-1.025)$ & $0.408(0.195-0.853)$ \\
\hline rs35285779 & B vs. A & PB & 2 & 0.172 & 0.034 & 0.17 & $0.691(0.442-1.08)$ & $0.711(0.519-0.975)$ \\
\hline rs35285779 & BB vs. AA & PB & 2 & 0.352 & 0.145 & 0.725 & $0.452(0.147-1.388)$ & $0.443(0.148-1.323)$ \\
\hline rs35285779 & BA vs. AA & PB & 2 & 0.434 & 0.102 & 0.51 & $0.741(0.517-1.062)$ & $0.741(0.517-1.062)$ \\
\hline rs35285779 & $\mathrm{BB}+\mathrm{BA}$ vs. $\mathrm{AA}$ & PB & 2 & 0.257 & 0.057 & 0.285 & $0.702(0.467-1.054)$ & $0.713(0.504-1.01)$ \\
\hline rs35285779 & $B B$ vs. $B A+A A$ & PB & 2 & 0.393 & 0.197 & 0.985 & $0.498(0.163-1.52)$ & $0.488(0.164-1.452)$ \\
\hline rs35285779 & B vs. A & $H B$ & 2 & 0.041 & 0.103 & 0.515 & $0.508(0.226-1.145)$ & $0.535(0.365-0.783)$ \\
\hline rs35285779 & BB vs. AA & $H B$ & 2 & 0.131 & 0.025 & 0.125 & $0.262(0.028-2.443)$ & $0.317(0.116-0.868)$ \\
\hline rs35285779 & BA vs. AA & $\mathrm{HB}$ & 2 & 0.161 & 0.031 & 0.155 & $0.592(0.305-1.149)$ & $0.598(0.375-0.954)$ \\
\hline rs35285779 & $\mathrm{BB}+\mathrm{BA}$ vs. $\mathrm{AA}$ & $H B$ & 2 & 0.081 & 0.104 & 0.52 & $0.519(0.236-1.144)$ & $0.535(0.344-0.833)$ \\
\hline rs35285779 & $B B$ vs. $B A+A A$ & $H B$ & 2 & 0.16 & 0.043 & 0.215 & $0.311(0.041-2.355)$ & $0.354(0.13-0.967)$ \\
\hline rs35285779 & B vs. A & Lung Cancer & 2 & 0.172 & 0.034 & 0.17 & $0.691(0.442-1.08)$ & $0.711(0.519-0.975)$ \\
\hline rs35285779 & $B B$ vs. $A A$ & Lung Cancer & 2 & 0.352 & 0.145 & 0.725 & $0.452(0.147-1.388)$ & $0.443(0.148-1.323)$ \\
\hline rs35285779 & BA vs. AA & Lung Cancer & 2 & 0.434 & 0.102 & 0.51 & $0.741(0.517-1.062)$ & $0.741(0.517-1.062)$ \\
\hline rs35285779 & $\mathrm{BB}+\mathrm{BA}$ vs. $\mathrm{AA}$ & Lung Cancer & 2 & 0.257 & 0.057 & 0.285 & $0.702(0.467-1.054)$ & $0.713(0.504-1.01)$ \\
\hline rs35285779 & $B B$ vs. $B A+A A$ & Lung Cancer & 2 & 0.393 & 0.197 & 0.985 & $0.498(0.163-1.52)$ & $0.488(0.164-1.452)$ \\
\hline rs7219582 & B vs. A & Overall & 4 & 0.386 & 0.077 & 0.385 & $0.822(0.645-1.048)$ & $0.82(0.659-1.021)$ \\
\hline rs7219582 & BA vs. AA & Overall & 4 & 0.035 & 0.538 & 1.000 & $0.75(0.3-1.873)$ & $0.491(0.344-0.7)$ \\
\hline rs7219582 & $B B+B A$ vs. $A A$ & Overall & 4 & 0.045 & 0.538 & 1.000 & $0.758(0.313-1.833)$ & $0.51(0.358-0.727)$ \\
\hline rs7219582 & B vs. A & Lung Cancer & 2 & 0.941 & 0.041 & 0.205 & $0.789(0.629-0.99)$ & $0.789(0.629-0.99)$ \\
\hline rs7219582 & BA vs. AA & Lung Cancer & 2 & 0.399 & $<0.001$ & $<0.001^{*}$ & $0.394(0.265-0.586)$ & $0.394(0.265-0.585)$ \\
\hline rs7219582 & $B B+B A$ vs. $A A$ & Lung Cancer & 2 & 0.436 & $<0.001$ & $<0.001^{*}$ & $0.414(0.278-0.614)$ & $0.413(0.278-0.614)$ \\
\hline rs9915936 & B vs. A & Overall & 4 & 0.873 & 0.038 & 0.19 & $0.708(0.51-0.981)$ & $0.707(0.51-0.981)$ \\
\hline rs9915936 & BA vs. AA & Overall & 4 & 0.668 & 0.014 & 0.07 & $0.634(0.44-0.914)$ & $0.633(0.44-0.91)$ \\
\hline rs9915936 & $B B+B A$ vs. $A A$ & Overall & 4 & 0.775 & 0.021 & 0.105 & $0.662(0.466-0.94)$ & $0.661(0.466-0.939)$ \\
\hline rs9915936 & B vs. A & PB & 2 & 0.806 & 0.034 & 0.17 & $0.667(0.459-0.971)$ & $0.667(0.459-0.97)$ \\
\hline rs9915936 & BA vs. AA & $\mathrm{PB}$ & 2 & 0.548 & 0.009 & $0.045^{*}$ & $0.567(0.369-0.871)$ & $0.566(0.369-0.87)$ \\
\hline rs9915936 & $\mathrm{BB}+\mathrm{BA}$ vs. $\mathrm{AA}$ & PB & 2 & 0.669 & 0.016 & 0.08 & $0.607(0.404-0.913)$ & $0.607(0.404-0.912)$ \\
\hline rs9915936 & B vs. A & $H B$ & 2 & 0.619 & 0.646 & 1.000 & $0.855(0.436-1.677)$ & $0.854(0.436-1.674)$ \\
\hline rs9915936 & BA vs. AA & $H B$ & 2 & 0.608 & 0.637 & 1.000 & $0.848(0.425-1.692)$ & $0.847(0.425-1.689)$ \\
\hline rs9915936 & $B B+B A$ vs. $A A$ & $H B$ & 2 & 0.608 & 0.637 & 1.000 & $0.848(0.425-1.692)$ & $0.847(0.425-1.689)$ \\
\hline rs9915936 & B vs. A & Lung Cancer & 2 & 0.806 & 0.034 & 0.17 & $0.667(0.459-0.971)$ & $0.667(0.459-0.97)$ \\
\hline rs9915936 & BA vs. AA & Lung Cancer & 2 & 0.548 & 0.009 & $0.045^{*}$ & $0.567(0.369-0.871)$ & $0.566(0.369-0.87)$ \\
\hline
\end{tabular}


Table 2 Results of pooled analysis for AXIN2 Polymorphism and cancer susceptibility (Continued)

\begin{tabular}{lllllllll}
\hline Polymorphism & Comparison & Subgroup & $\mathbf{N}$ & $\mathbf{P}_{\mathbf{H}}$ & $\mathbf{P}_{\mathbf{Z}}$ & $\mathbf{P}_{\text {Adjust }}$ & OR \& 95\%Cl (Random) & OR \& 95\%Cl (Fixed) \\
\hline rs9915936 & BB + BA vs. AA & Lung Cancer & 2 & 0.669 & 0.016 & 0.08 & $0.607(0.404-0.913)$ & $0.607(0.404-0.912)$ \\
\hline$P_{H} P$
\end{tabular}

$P_{H} P$ value of $Q$ test for heterogeneity test, $P_{Z} P$ value of meta-analysis, $P_{\text {Adjust }}$ Adjust $P_{Z}$ value by Bonferroni corrections, $P_{A d j u s t}=P_{Z} * 5, P-B$ Population based, $H W E$ Hardy Weinberg Equilibrium, $Y$ polymorphisms conformed to HWE in the control group, $N$ polymorphisms didn't conform to HWE in the control group

* $P$ value less than 0.05 was considered as statistically significant

rs35285779, it was revealed significant association with overall cancer risk in $\mathrm{BA}$ vs. $\mathrm{AA}$ and $\mathrm{BB}+\mathrm{BA}$ vs. $\mathrm{AA}$ models (PAdjust<0.05) (Table 2, Figure S4). For rs7219582, it showed significant relationship with lung cancer risk in $\mathrm{BA}$ vs. $\mathrm{AA}$ and $\mathrm{BB}+\mathrm{BA}$ vs. AA models (PAdjust<0.05) (Table 2, Figure S10). For rs9915936, which also informed significant association with risk of $\mathrm{PB}$ source and lung cancer in BA vs. AA model (PAdjust< 0.05), respectively (Table 2, Figure S12). As to rs2240307, rs35415678, rs3923086, rs3923087, rs4072245, rs4791171 and rs7224837 polymorphisms, the pooled analysis data didn't show any correlation with cancers, not only in overall risk, but also in cancer type, ethnicity or source of control (Table S2, Figure S3, S5, S6, S7, S8, S9, S11).

\section{Sensitivity analysis and publication bias}

To check the influence of individual study on overall data, we applied sensitivity analysis, and the results of the pooled analysis proved that the OR value was not influenced by individual study (Fig. 3, S13 and Table S3). At the same time, to evaluate the publication bias, Begg's funnel plot and Egger's test were performed, and the results didn't show asymmetric evidence (Fig. 4, S14 and Table S4).

\section{Linkage disequilibrium (LD) analysis of $A X I N-2$ polymorphisms}

LD analysis was assessed to evaluate the inner interaction of each $A X I N-2$ polymorphism and the results were shown in Fig. 5. Obviously, there was significant LD between rs7224837 and rs7210356 in CEU populations $\left(r^{2}=0.91\right)$, the same as between $r s 7210356$ and $r s 9915936\left(r^{2}=0.91\right), \quad r s 1133683$ and $r s 4791171\left(r^{2}=\right.$ $0.85), r s 35415678$ and $r s 35285779\left(r^{2}=0.84\right)$. There was significant LD between $r s 7224837$ and $r s 9915936$ in CHB\&CHS populations $\left(r^{2}=0.93\right)$, the same as between $r s 1133683$ and $r s 4791171\left(r^{2}=0.93\right), \quad r s 1133683$ and $r s 3923087\left(r^{2}=0.83\right)$. There was significant LD between

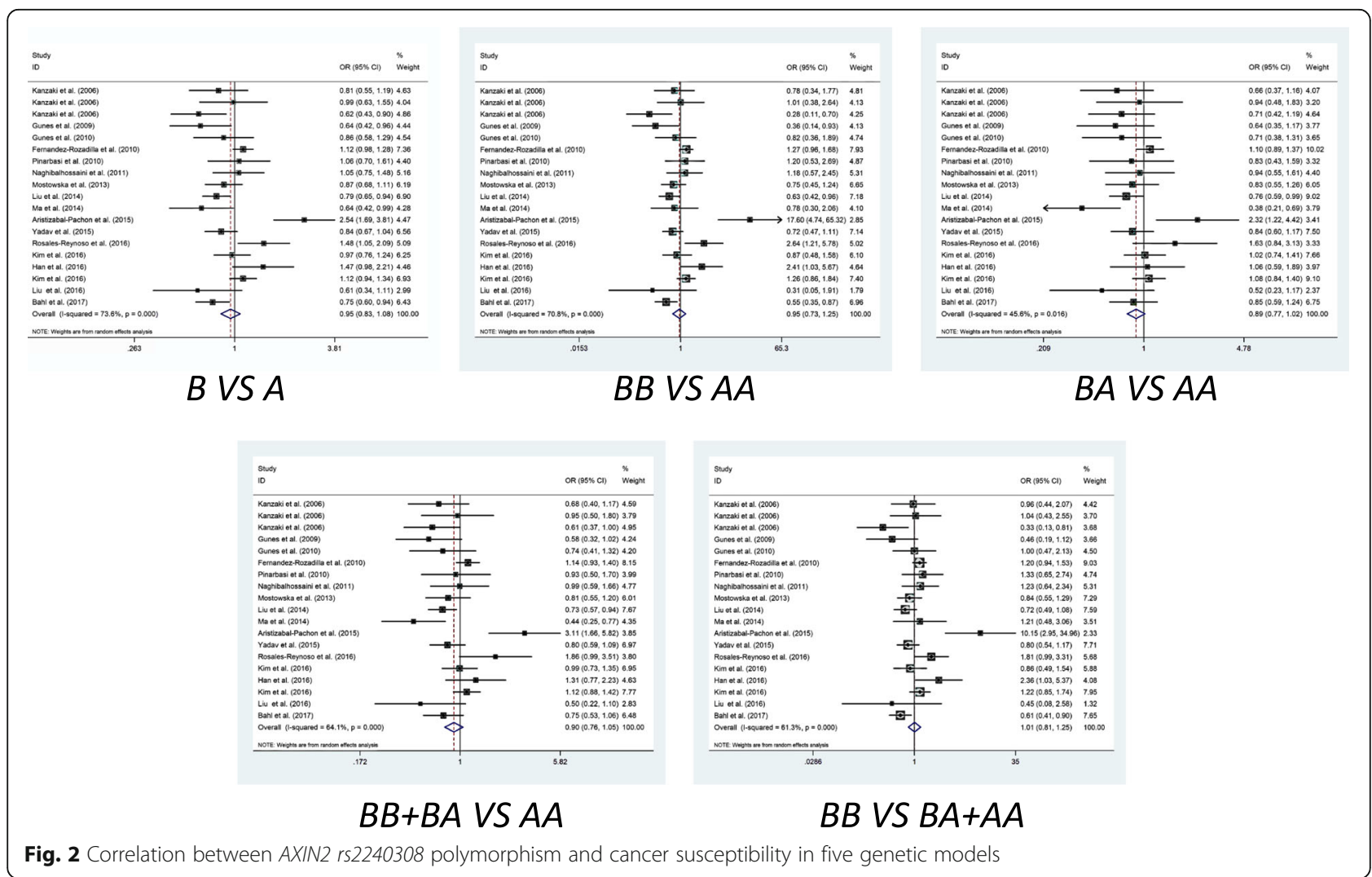



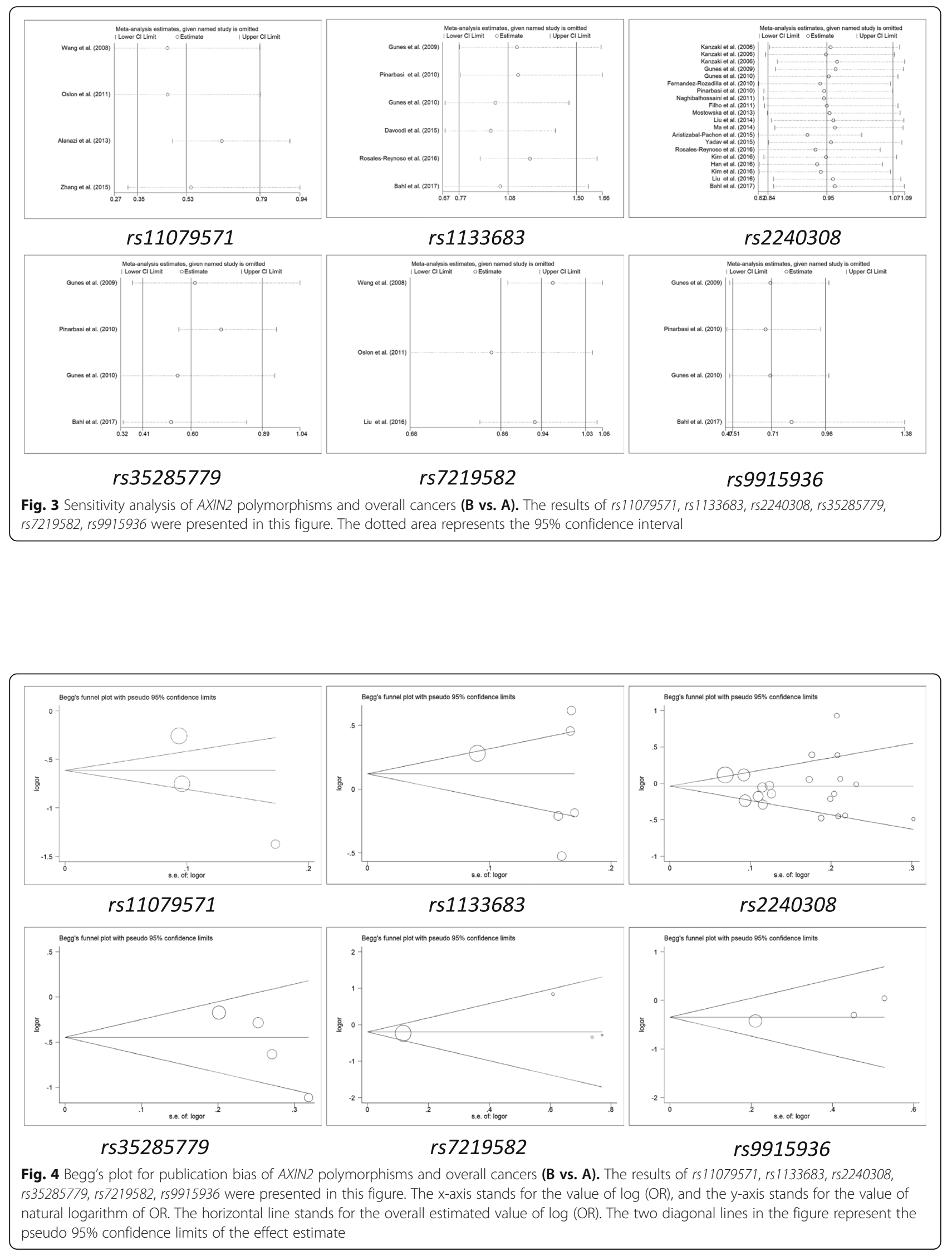


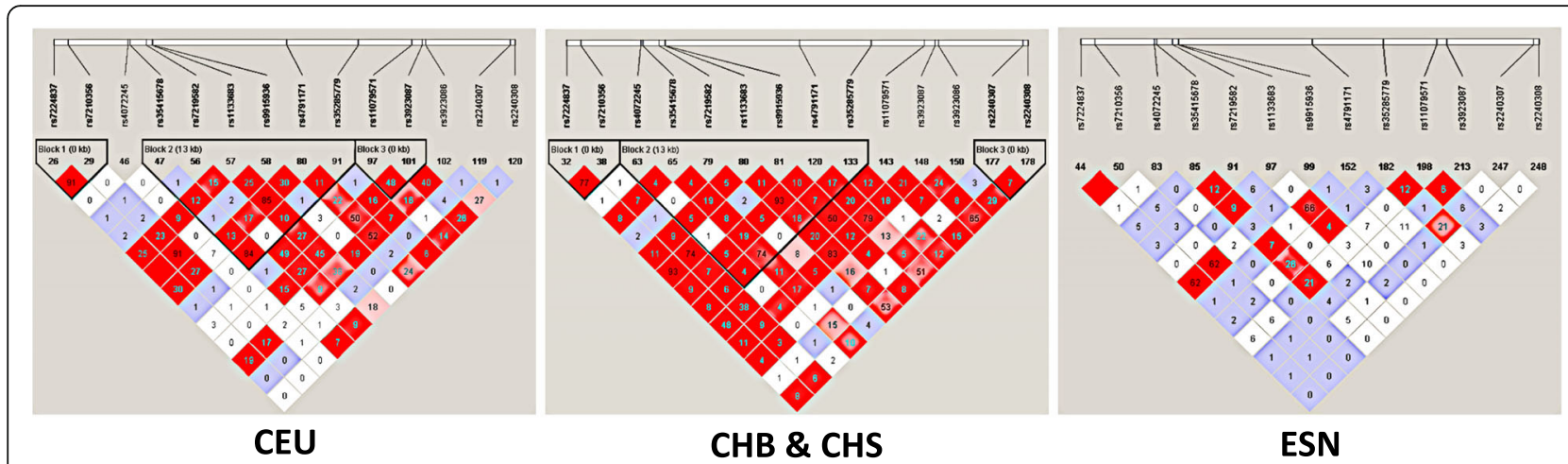

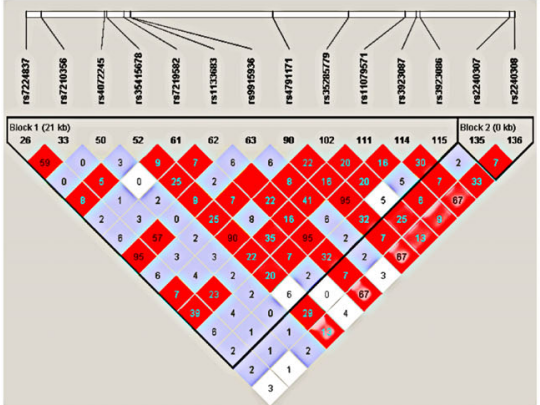

JPT

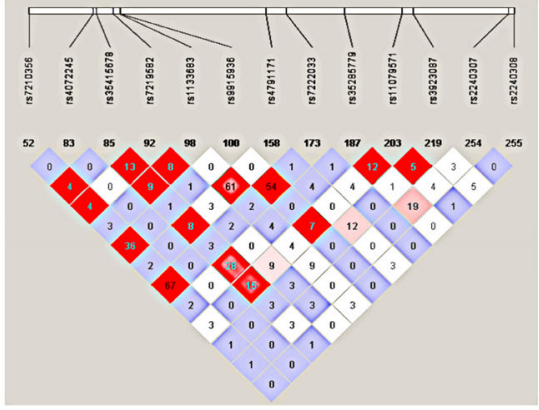

YRI

Fig. 5 LD analysis for AXIN-2 polymorphisms in different populations acquired from 1000 Genomes Project. The value of $r^{2}$ is showed in each square, and white colors represent no significant LD between different polymorphisms. CEU: Utah residents with Northern and Western European ancestry from the CEPH collection; CHB: Han Chinese in Beijing, China; CHS: Southern Han Chinese, China; ESN: Esan in Nigeria; JPT: Japanese in Tokyo, Japan; YRI: Yoruba in Ibadan, Nigeria

rs7224837 and $r s 9915936$ in ESN populations $\left(r^{2}=0.62\right)$, the same as between $r s 7210356$ and $r s 9915936\left(r^{2}=\right.$ $0.62), r s 1133683$ and $r s 4791171\left(r^{2}=0.66\right)$. There was significant LD between $r s 7224837$ and $r s 9915936$ in JPT populations $\left(r^{2}=0.95\right)$, the same as between $r s 35415678$ and $r s 35285779\left(r^{2}=0.90\right)$, $r s 1133683$ and $r s 3923087$ $\left(r^{2}=0.95\right), r s 4791171$ and $r s 3923087\left(r^{2}=0.95\right)$. There was significant LD between $r s 7210356$ and $r s 7222033$ in YRI populations $\left(r^{2}=0.67\right)$, the same as between $r s 9915936$ and $r s 7222033\left(r^{2}=0.54\right)$.

\section{Discussion}

AXIN2 plays an important role as a negative regulator in regulating $\beta$-catenin stability. As $\beta$-catenin was well studied as an important gene related to many cancers [47-50], the correlation between AXIN2 and tumor progression and metastasis have also been well reported by many studies in the past few decades. Xie et al. [51] reported AXIN2 can be targeted by miR143HG/miR-1275 to regulate breast cancer progression by modulating the Wnt/ $\beta$-catenin pathway. Ren et al. [52] revealed that AXIN2 was a target of miR-454-3p and was involved in the activation of $\mathrm{Wnt} / \beta$-catenin signaling, which can be suppressed by miR-454-3p to promote metastasis and the stemness of breast cancer. Chen et al. [11] demonstrated that AXIN2 could be down-regulated by miR-544, thus to promote human osteosarcoma cell proliferation. Lu et al. [53] reported that AXIN2 was identified to be a functional downstream target of miR-374a, and decreased expression of Axin 2 could promote OS cell proliferation.

Previous studies have also demonstrated the association between AXIN2 and cancer risk and susceptibility. Liu et al. [15] reported that AXIN2 rs11655966 and rs3923086 polymorphism had significant associations with papillary thyroid carcinoma. Aristizabal-Pachon et al. [42] showed significant association between AXIN2 rs151279728 and rs2240308 polymorphisms and breast cancer susceptibility. Ma et al. [40] concluded that there was a significant correlation between rs2240308 polymorphism and the susceptibility of prostate cancer, while E.Pinarbasi et al. [16] reported that there was no significant correlation between prostate cancer susceptibility and rs2240308 polymorphism in Turkish population.

Judge from the studies related to AXIN2 polymorphism and cancer risk and susceptibility, the results seem not consistent. So, we preformed this meta-analysis to the current evidence for AXIN2 polymorphism to cancer risk. As the results showed in Figures and Tables, we 
concluded that AXIN2 rs11079571 had significant correlation with overall cancers and Asian population subtype. As for other polymorphisms, like rs1133683 and rs35285779 had significant correction with overall cancers in two genetic models (rs1133683, BB vs. AA and $\mathrm{BB}$ vs. $\mathrm{BA}+\mathrm{AA})(r s 35285779, \mathrm{BA}$ vs. $\mathrm{AA}$ and $\mathrm{BB}+\mathrm{BA}$ vs. AA), however, the others had no strong relationship with overall cancer risk. As to subtype cancers, rs11079571 showed significant correlation with breast cancer, rs1133683, rs7219582 and rs9915936 indicated significant correlation with lung cancer. What's more, the LD analysis showed a significant LD between rs7224837 and rs7210356/rs9915936, as well as between rs9915936 and rs7210356/rs7224837, which means that maybe we should combine two or more polymorphisms to analysis the correlation between AXIN2 and cancer risk and susceptibility in future.

At the same time, we must realize the limitations that exist in this study. Firstly, an enlarged numbers of articles that involved are needed in the analysis, especially for AXIN2 rs7224837 polymorphism. Secondly, when we searched the articles, we only involved the studies in English and Chinese, which may also cause bias for not involving other languages. Thirdly, for subtype analysis, we didn't analyze every cancer for each polymorphism, which may lead to some shortcomings. Fourthly, geneenvironment interactions were ignored in this study because of lack necessary data.

\section{Conclusions}

In conclusion, our updated study suggests that AXIN2 rs11079571, rs1133683 and rs35285779 polymorphisms are associated with overall cancer susceptibility, which may provide a new insight to understand the correlation between AXIN2 gene and cancer risk. What's more, the combination of two or more polymorphisms may benefit us to better understand the function of AXIN2 polymorphisms in different populations. Future large scale and well-designed research are required to validate these effects in more detail.

\section{Abbreviations}

LD: Linkage disequilibrium; HWE: Hardy-Weinberg equilibrium; NOS: Newcastle-Ottawa Scale; PB: Population based; CHS: Southern Han Chinese, China; CHB: Han Chinese in Beijing, China; ESN: Esan in Nigeria; YRI: Yoruba in Ibadan, Nigeria; JPT: Japanese in Tokyo, Japan; CRC: Colorectal Cancer; PTC: Papillary Thyroid Carcinoma

\section{Supplementary Information}

The online version contains supplementary material available at https://doi. org/10.1186/s12885-021-08092-0.

Additional file 1 : Table S1. Methodological quality of the included studies according to the Newcastle-Ottawa Scale. Table S2. Results of pooled analysis for AXIN2 Polymorphism and cancer susceptibility. Table S3. Details of the sensitivity analyses for AXIN2 polymorphism and urinary cancer risk. Table S4. $P$ values of the Egger's test for AXIN2 polymorphism.

Additional file 2 : Figure S1. Meta-analysis ofAXIN2-rs1 1079571 polymorphism and overall cancer risk in 5 genetic models.

Additional file 3 : Figure S2. Meta-analysis ofAXIN2-rs1133683 polymorphism and overall cancer risk in 5 genetic models.

Additional file 4 : Figure S3. Meta-analysis ofAXIN2-rs2240307 polymorphism and overall cancer risk in 3 genetic models.

Additional file $\mathbf{5}$ : Figure S4. Meta-analysis ofAXIN2-rs35285779 polymorphism and overall cancer risk in 5 genetic models.

Additional file 6 : Figure S5. Meta-analysis ofAXIN2-rs35415678 polymorphism and overall cancer risk in 3 genetic models.

Additional file 7 : Figure S6. Meta-analysis ofAXIN2-rs3923086 polymorphism and overall cancer risk in 5 genetic models.

Additional file 8 : Figure S7. Meta-analysis ofAXIN2-rs3923087 polymorphism and overall cancer risk in 5 genetic models.

Additional file 9 : Figure S8. Meta-analysis ofAXIN2-rs4072245 polymorphism and overall cancer risk in 3 genetic models.

Additional file 10 : Figure S9. Meta-analysis ofAXIN2-rs4791171 polymorphism and overall cancer risk in 5 genetic models.

Additional file 11 : Figure S10. Meta-analysis ofAXIN2-rs7219582 polymorphism and overall cancer risk in 5 genetic models.

Additional file 12 : Figure S11. Meta-analysis of AXIN2-rs7224837 polymorphism and overall cancer risk in 5 genetic models.

Additional file 13 : Figure S12. Meta-analysis ofAXIN2-rs9915936 polymorphism and overall cancer risk in 5 genetic models.

Additional file 14 : Figure S13. Sensitivity analysis ofAXIN2 polymorphism and overall cancer (Bvs.A). The results of rs2240307, rs35415678, rs3923086, rs3923087, rs4072245, rs4791171, rs7210356, rs 7224837 were presented in this figure. The dotted area represents the 95\% confidence interval.

Additional file 15 : Figure S14. Begg'splot ofAXIN2 polymorphism and overall cancer (Bvs.A). The results of rs2240307, rs35415678, rs3923086, rs3923087, rs4072245, rs4791171, rs7224837 were presented in this figure. The $x$-axis stands for the value of $\log (\mathrm{OR})$, and the $y$-axis stands for the value of natural logarithm of OR. The horizontal line stands for the overall estimated value of $\log (\mathrm{OR})$. The two diagonal lines in the figure represent the pseudo $95 \%$ confidence limits of the effect estimate.

\section{Acknowledgements}

We thank Thelma for editing the manuscript.

\section{Authors' contributions}

$G L$ and $W W$ ensured the integrity of the entire study. $X L$ and $Y L$ performed the whole experiments and was a major contributor in writing the manuscript. GL and $X L$ were in charge of data analysis. $Y L, G L$ and $W W$ were responsible for revising the manuscript and checking all data. All authors read and approved the final manuscript.

Funding

Not applicable.

Availability of data and materials

All data are presented in the figures, tables and supplementary files.

\section{Declarations}

Ethics approval and consent to participate Not applicable.

Consent for publication

Not applicable.

Competing interests

The authors declare that they have no competing interests. 


\section{Author details}

'Department of Geriatric Surgery, Xiangya Hospital, Central South University, Changsha 410008, China. ${ }^{2}$ National Clinical Research Center for Geriatric Disorders, Xiangya Hospital, Central South University, Changsha 410008, China. ${ }^{3}$ Department of General Surgery, Xiangya Hospital, Central South University, Changsha 410008, China.

Received: 22 September 2020 Accepted: 23 March 2021

Published online: 01 April 2021

\section{References}

1. Siegel RL, Miller KD, Jemal A. Cancer statistics, 2019. CA Cancer J Clin. 2019; 69:7-34.

2. Chen W, Zheng R, Baade PD, Zhang S, Zeng H, Bray F, et al. Cancer statistics in China, 2015. CA Cancer J Clin. 2016;66(2):115-32. https://doi.org/10.3322/ caac.21338.

3. Ferlay J, Colombet M, Soerjomataram I, Dyba T, Randi G, Bettio M, et al. Cancer incidence and mortality patterns in Europe: estimates for 40 countries and 25 major cancers in 2018. Eur J Cancer. 2018;103:356-87. https://doi.org/10.1016/..ejca.2018.07.005.

4. Hongdan L, Feng L. miR-3120-5p promotes colon cancer stem cell stemness and invasiveness through targeting Axin2. Biochem Biophys Res Commun. 2018;496(2):302-8. https://doi.org/10.1016/j.bbrc.2018.01.021.

5. Amalia R, Abdelaziz M, Puteri MU, Hwang J, Anwar F, Watanabe Y, et al. TMEPAI/PMEPA1 inhibits Wnt signaling by regulating $\beta$-catenin stability and nuclear accumulation in triple negative breast cancer cells. Cell Signal. 2019; 59:24-33. https://doi.org/10.1016/j.cellsig.2019.03.016.

6. Zhang X, Kim K, Zheng Z, et al. Snail and Axin2 expression predict the malignant transformation of oral leukoplakia. Oral Oncol. 2017;73:48-55. https://doi.org/10.1016/j.oraloncology.2017.08.004.

7. Li Y, Jin K, van Pelt GW, van Dam H, Yu X, Mesker WE, et al. C-Myb enhances breast cancer invasion and metastasis through the $\mathrm{Wnt} / \mathrm{\beta}$ catenin/Axin2 pathway. Cancer Res. 2016;76(11):3364-75. https://doi.org/1 0.1158/0008-5472.CAN-15-2302

8. Dong X, Seelan RS, Qian C, Mai M, Liu W. Genomic structure, chromosome mapping and expression analysis of the human AXIN2 gene. Cytogenet Genome Res. 2001;93(1-2):26-8. https://doi.org/10.1159/000056942.

9. Mai M, Qian C, Yokomizo A, Smith DI, Liu W. Cloning of the human homolog of conductin (AXIN2), a gene mapping to chromosome 17q23q24. Genomics. 1999;55(3):341-4. https://doi.org/10.1006/geno.1998.5650.

10. Chen D, Li SG, Chen JY, Xiao M. MiR-183 maintains canonical Wnt signaling activity and regulates growth and apoptosis in bladder cancer via targeting AXIN2. Eur Rev Med Pharmacol Sci. 2018;22(15):4828-36. https://doi.org/10.2 6355/eurrev_201808_15618.

11. Chen $M$, Liu YY, Zheng MQ, Wang XL, Gao XH, Chen L, et al. microRNA-544 promoted human osteosarcoma cell proliferation by downregulating AXIN2 expression. Oncol Lett. 2018;15(5):7076-82. https://doi.org/10.3892/ol.2018. 8218.

12. Kim WK, Byun WS, Chung $\mathrm{H}$, et al. Esculetin suppresses tumor growth and metastasis by targeting Axin2/E-cadherin axis in colorectal cancer. Biochem Pharmacol. 2018;152:71-83. https://doi.org/10.1016/j.bcp.2018.03.009.

13. Otero L, Lacunza E, Vasquez V, Arbelaez V, Cardier F, González F. Variations in AXIN2 predict risk and prognosis of colorectal cancer. BDJ Open. 2019; 5(1):13. https://doi.org/10.1038/s41405-019-0022-z.

14. Zhong A, Pan X, Shi M, Xu H. $148 \mathrm{C} / \mathrm{T}$ polymorphism of Axin2 contributes to a decreased risk of cancer: evidence from a meta-analysis. Onco Targets Ther. 2015;8:1957-66. https://doi.org/10.2147/OTT.S86738.

15. Liu X, Li S, Lin X, Yan K, Zhao L, Yu Q, et al. AXIN2 is associated with papillary thyroid carcinoma. Iran Red Crescent Med J. 2016;18(2):e20960. https://doi.org/10.5812/ircmj.20960.

16. Pinarbasi E, Gunes EG, Pinarbasi H, Donmez G, Silig Y. AXIN2 polymorphism and its association with prostate cancer in a Turkish population. Med Oncol. 2011;28(4):1373-8. https://doi.org/10.1007/s12032-010-9588-y.

17. Xu B, Yuan W, Shi L, Zuo L, Wu XY, Zhang W, et al. New insights into the association between AXIN2 $148 \mathrm{C} / \mathrm{T}, 1365 \mathrm{C} / \mathrm{T}$, and rs4791171 A/G variants and cancer risk. Cancer Cell Int. 2019;19(1):119. https://doi.org/10.1186/s1293 5-019-0840-z.

18. Dai F, Zhu L, Zhang W, et al. The association between three AXIN2 variants and cancer risk. J Cell Biochem. 2019;120(9):15561-71. https://doi.org/10.1 002/jcb.28823.
19. Bonferroni CE. Teoria Statistica Delle Classi e Calcolo Delle Probabilità. Comm Firenze. 1936;1936:216-8.

20. DerSimonian R, Laird N. Meta-analysis in clinical trials. Control Clin Trials. 1986;7(3):177-88. https://doi.org/10.1016/0197-2456(86)90046-2.

21. Yu Y, Tao Y, Liu L, Yang J, Wang L, Li X, et al. New concept of the Axin2 rs2240308 polymorphism and cancer risk: an updated meta-analysis Neoplasma. 2017;64(2):269-77. https://doi.org/10.4149/neo_2017_214.

22. Thakkinstian A, McElduff $P, D^{\prime} E s t e ~ C$, et al. A method for meta-analysis of molecular association studies. Stat Med. 2005;24(9):1291-306. https://doi. org/10.1002/sim.2010.

23. Begg CB, Mazumdar M. Operating characteristics of a rank correlation test for publication bias. Biometrics. 1994;50(4):1088-110. https://doi.org/10.23 07/2533446.

24. Higgins JP, Thompson SG. Quantifing heterogeneity in a meta-analysis. Stat Med. 2002;21(11):1539-58. https://doi.org/10.1002/sim.1186.

25. Wang X, Goode EL, Fredericksen ZS, Vierkant RA, Pankratz VS, Liu-Mares W, et al. Association of genetic variation in genes implicated in the $\beta$-catenin destruction complex with risk of breast cancer. Cancer Epidemiol Biomark Prev. 2008;17(8):2101-8. https://doi.org/10.1158/1055-9965.EPI-08-0134.

26. Alanazi MS, Parine NR, Shaik JP, Alabdulkarim HA, Ajaj SA, Khan Z. Association of single nucleotide polymorphisms in Wnt signaling pathway genes with breast cancer in Saudi patients. PLoS One. 2013;8(3):e59555. https://doi.org/10.1371/journal.pone.0059555.

27. Zhang L, Zhou J, Ye Y, et al. Genetic polymorphisms in Wnt signaling pathway and acute leukemia. J Sichuan Univ (Med Sci Edi). 2015;46(3):4038.

28. Gunes EG, Pinarbasi E, Pinarbasi H, Silig Y. Strong association between lung cancer and the AXIN2 polymorphism. Mol Med Rep. 2009;2(6):1029-35. https://doi.org/10.3892/mmr_00000210.

29. Bahl C, Sharma S, Singh N, et al. Association study between genetic variations in Axin2 gene and lung cancer risk in north Indian population: a multiple interaction analysis. Tumor Biol. 2017;39(4):568836143.

30. Gunes EG, Pinarbasi E, Pinarbasi H. AXIN2 polymorphism and its association with astrocytoma in a Turkish population. Mol Med Rep. 2010;3(4):705-9. https://doi.org/10.3892/mmr_00000321.

31. Mostowska A, Pawlik P, Sajdak S, et al. An analysis of polymorphisms within the Wnt signaling pathway in relation to ovarian cancer risk in a polish population. Mol Diagn Ther. 2013;18(1):85-91.

32. Rosales-Reynoso MA, Arredondo-Valdez AR, Wence-Chávez LI, Barros-Núñez P. Gallegos-Arreola MP, Flores-Martínez SE, et al. AXIN2 polymorphisms and their association with colorectal cancer in Mexican patients. Genet Test Mol Biomarkers. 2016;20(8):438-44. https://doi.org/10.1089/gtmb.2016.0026.

33. Han S, Lv L, Wang X, et al. Association of AXIN2 and MMP7 polymorphisms with non-small cell lung cancer in Chinese Han population. Int J Clin Exp Pathol. 2016;9(2):2253-8.

34. Andrade Filho PA, Letra A, Cramer A, Prasad JL, Garlet GP, Vieira AR, et al. Insights from studies with oral cleft genes suggest associations between WNT-pathway genes and risk of oral cancer. J Dent Res. 2011;90(6):740-6. https://doi.org/10.1177/0022034511401622.

35. Kanzaki H, Ouchida M, Hanafusa $H$, Yano M, Suzuki H, Aoe M, et al. Single nucleotide polymorphism of the AXIN2 gene is preferentially associated with human lung cancer risk in a Japanese population. Int J Mol Med. 2006; 18(2):279-84.

36. Liu D, Li L, Yang Y, Liu W, Wu J. The Axin2 rs2240308 polymorphism and susceptibility to lung cancer in a Chinese population. Tumor Biol. 2014; 35(11):10987-91. https://doi.org/10.1007/s13277-014-2399-6.

37. Kim CH. Environmental tobacco smoke, genetic susceptibility, and lung cancer among never smokers: ULCA; 2016. ProQuest ID: Kim_UCLA_0031D_ 14561. Merritt ID: ark:/13030/m5xt0h1p. Retrieved from https://escholarship. org/uc/item/81j69493

38. Fernández-Rozadilla C, de Castro L, Clofent J, Brea-Fernández A, Bessa X, Abulí $A$, et al. Single nucleotide polymorphisms in the Wnt and BMP pathways and colorectal cancer risk in a Spanish cohort. PLoS One. 2010; 5(9):e12673. https://doi.org/10.1371/journal.pone.0012673.

39. Naghibalhossaini F, Zamani M, Mokarram P, Khalili I, Rasti M, Mostafavi-pour Z. Epigenetic and genetic analysis of WNT signaling pathway in sporadic colorectal cancer patients from Iran. Mol Biol Rep. 2012;39(5):6171-8. https://doi.org/10.1007/s11033-011-1434-6.

40. Ma C, Liu C, Huang P, et al. Significant association between the Axin2 rs2240308 single nucleotide polymorphism and the incidence of prostate cancer. Oncol Lett. 2014;8(2):789-94. https://doi.org/10.3892/ol.2014.2177. 
41. Mostowska A, Pawlik P, Sajdak S, Markowska J, Pawałowska M, Lianeri M, et al. An analysis of polymorphisms within the Wnt signaling pathway in relation to ovarian cancer risk in a polish population. Mol Diagn Ther. 2014; 18(1):85-91. https://doi.org/10.1007/s40291-013-0059-y.

42. Aristizabal-Pachon AF, Carvalho TI, Carrara HH, Andrade J, Takahashi CS. AXIN2 polymorphisms, the $\beta$-catenin destruction complex expression profile and breast cancer susceptibility. Asian Pac J Cancer Prev. 2015;16(16):727784. https://doi.org/10.7314/APJCP.2015.16.16.7277.

43. Yadav A, Gupta A, Yadav S, Rastogi N, Agrawal S, Kumar A, et al. Association of Wnt signaling pathway genetic variants in gallbladder cancer susceptibility and survival. Tumor Biol. 2016;37(6):8083-95. https://doi.org/1 0.1007/s13277-015-4728-9.

44. Kim SS, Cho HJ, Lee H, et al. Genetic polymorphisms in the Wnt/ß-catenin pathway genes as predictors of tumor development and survival in patients with hepatitis B virus-associated hepatocellular carcinoma. Clin Biochem. 2016;49(10-11):792-801. https://doi.org/10.1016/j.clinbiochem.2016.01.025.

45. Parine NR, Azzam NA, Shaik J, Aljebreen AM, Alharbi O, Almadi MA, et al. Genetic variants in the WNT signaling pathway are protectively associated with colorectal cancer in a Saudi population. Saudi J Biol Sci. 2019;26(2): 286-93. https://doi.org/10.1016/j.sjbs.2018.05.018.

46. Pierzynski JA, Hildebrandt MA, Kamat AM, Lin J, Ye Y, Dinney CPN, et al. Genetic variants in the Wnt/ $\beta$-catenin signaling pathway as indicators of bladder cancer risk. J Urol. 2015;194(6):1771-6. https://doi.org/10.1016/j. juro.2015.07.032.

47. Al-Dhfyan A, Alhoshani A, Korashy HM. Aryl hydrocarbon receptor/ cytochrome P450 1A1 pathway mediates breast cancer stem cells expansion through PTEN inhibition and $\beta$-catenin and Akt activation. Mol Cancer. 2017;16(1):14. https://doi.org/10.1186/s12943-016-0570-y.

48. Persad S, Troussard AA, McPhee TR, et al. Tumor suppressor PTEN inhibits nuclear accumulation of beta-catenin and $T$ cell/lymphoid enhancer factor 1-mediated transcriptional activation. J Cell Biol. 2001;153(6):1161-74. https://doi.org/10.1083/jcb.153.6.1161.

49. Ma J, Guo X, Zhang J, Wu D, Hu X, Li J, et al. PTEN gene induces cell invasion and migration via regulating AKT/GSK-3 $\beta / \beta$-catenin signaling pathway in human gastric cancer. Dig Dis Sci. 2017;62(12):3415-25. https:// doi.org/10.1007/s10620-017-4764-y.

50. Ge H, Liang C, Li Z, An D, Ren S, Yue C, et al. DcR3 induces proliferation, migration, invasion, and EMT in gastric cancer cells via the PI3KAKT/GSK3//ß-catenin signaling pathway. Onco Targets Ther. 2018;11:4177-87. https://doi.org/10.2147/OTT.S172713.

51. Xie $H$, Huang $H$, Huang W, et al. LncRNA miR143HG suppresses bladder cancer development through inactivating Wnt/3-catenin pathway by modulating miR-1275/AXIN2 axis. J Cell Physiol. 2018;234(7):11156-64.

52. Ren L, Chen H, Song J, Chen X, Lin C, Zhang X, et al. MiR-454-3p-mediated Wnt/ $\beta$-catenin signaling antagonists suppression promotes breast cancer metastasis. Theranostics. 2019;9(2):449-65. https://doi.org/10.7150/thno.2 9055.

53. Lu T, Zhang $C$, Chai MX, An YB, Jia JL. MiR-374a promotes the proliferation of osteosarcoma cell proliferation by targeting Axin2. Int J Clin Exp Pathol. 2015;8(9):10776-83.

\section{Publisher's Note}

Springer Nature remains neutral with regard to jurisdictional claims in published maps and institutional affiliations.

\section{Ready to submit your research? Choose BMC and benefit from:}

- fast, convenient online submission

- thorough peer review by experienced researchers in your field

- rapid publication on acceptance

- support for research data, including large and complex data types

- gold Open Access which fosters wider collaboration and increased citations

- maximum visibility for your research: over $100 \mathrm{M}$ website views per year

At BMC, research is always in progress.

Learn more biomedcentral.com/submissions 